\title{
Horizontal circulation and jumps in Hamiltonian wave models
}

\author{
E. Gagarina ${ }^{1}$, J. van der $\operatorname{Vegt}^{1}$, and O. Bokhove ${ }^{1,2}$ \\ ${ }^{1}$ Department of Applied Mathematics, University of Twente, Enschede, the Netherlands \\ ${ }^{2}$ School of Mathematics, University of Leeds, Leeds, UK \\ Correspondence to: O. Bokhove (o.bokhove@leeds.ac.uk)
}

Received: 10 January 2013 - Revised: 16 May 2013 - Accepted: 17 May 2013 - Published: 12 July 2013

\begin{abstract}
We are interested in the modelling of wave-current interactions around surf zones at beaches. Any model that aims to predict the onset of wave breaking at the breaker line needs to capture both the nonlinearity of the wave and its dispersion. We have therefore formulated the Hamiltonian dynamics of a new water wave model, incorporating both the shallow water and pure potential flow water wave models as limiting systems. It is based on a Hamiltonian reformulation of the variational principle derived by Cotter and Bokhove (2010) by using more convenient variables. Our new model has a three-dimensional velocity field consisting of the full three-dimensional potential velocity field plus extra horizontal velocity components. This implies that only the vertical vorticity component is nonzero. Variational Boussinesq models and Green-Naghdi equations, and extensions thereof, follow directly from the new Hamiltonian formulation after using simplifications of the vertical flow profile. Since the full water wave dispersion is retained in the new model, waves can break. We therefore explore a variational approach to derive jump conditions for the new model and its Boussinesq simplifications.
\end{abstract}

\section{Introduction}

The beach surf zone is defined as the region of wave breaking and white capping between the moving shore line and the (generally time-dependent) breaker line. Let us consider wave propagation from deeper water to shallow water regions. The start of the surf zone on the offshore side is at the breaker line where sustained wave breaking begins. It demarcates the points where the nonlinearity of the waves becomes strong enough to outweigh dispersion. The waves thus start to overturn. From the point of breaking till the shore, the waves lose energy and generate vorticity. A mathemati- cal model that can predict the onset of wave breaking at the breaker line will need to capture both the nonlinearity of the waves and their dispersion. Moreover the model has to include vorticity effects to simulate wave-current interactions.

Various mathematical models are used to describe water waves. A popular model for smooth waves in deep water is the potential flow model, but its velocity field does not include vorticity. In the near-shore region, vorticity effects are, however, important. When obliquely incident waves shoal in shallow water, steepen and break, a horizontal shear or vertical vorticity is generated. On semi-enclosed or enclosed beaches, this leads to an overall circulation induced by wave breaking. A classical hydraulic model for the surf zone is the shallow water model. The complicated, turbulent threedimensional wave breaking is approximated in this model by discontinuities or so-called bores. These are special relations holding across the jumps connecting the right and left states of the flow. Mass and momentum are conserved across the discontinuity, while energy is not, as can be expected from observing the white capping zone of fine-scale splashes and sprays in the broken wave (Whitham, 1974). Shallow water waves are not dispersive, and these waves tend to break too early in comparison with real, dispersive waves. Boussinesq models include internal wave dispersion to a higher degree of accuracy, but dispersion always seems to beat nonlinearity. Therefore wave overturning tends to be prevented in these models. The variational Boussinesq model proposed by Klopman et al. (2010) could be a notable exception, but it is based on the ansatz of potential flow. In three dimensions, a purely potential-flow model cannot be extended by inclusion of bores and hydraulic jumps as a simple model to represent wave breaking. The reason is that at least some vorticity has to be generated by bores that have non-uniformities along their jump line as was shown by Pratt (1983), Peregrine (1998), and Peregrine and Bokhove (1998). 
We therefore seek to develop a more advanced model that includes both the shallow water approximation of breaking waves as bores and the accurate dispersion of the potential flow model. Such a model was obtained by Cotter and Bokhove (2010) from a parent Eulerian variational principle with extended Clebsch variables, in which the vortical parts only depended on the horizontal coordinates. This restricts the vorticity to have a vertical component only. Extended Clebsch variables may, however, be less convenient algebraically and also yield a larger phase space of variables. We therefore reformulate this system in terms of surface velocity, velocity potential and water depth, and derive the Hamiltonian structure including its Poisson bracket. This new water wave model can be reduced to the shallow water equations, the potential flow model, and the Boussinesq model of Klopman et al. (2010) under corresponding restrictions. The Green-Naghdi equations emerge from the variational Boussinesq model by introducing a parabolic potential flow profile in the Poisson bracket, as well as another, columnar approximation of the velocity in the Hamiltonian.

Finally, a new variational approach to derive jump conditions across bores is also explored. It is inspired by the work of Wakelin (1993) for stationary shock or jump conditions for the shallow water equations. These results have been extended to moving shocks in shallow water based on the variational principles for the relevant Clebsch variables. Naturally, this approach allows us to obtain jump conditions for the new water wave model as well. The jump relations can be implemented in any system with a variational and Hamiltonian structure, but not every system with a Hamiltonian structure allows shocks or discontinuities to persist in time. For example, it was shown by El et al. (2006) that the Green-Naghdi system has an unsteady undular bore, i.e. an initial discontinuity in the free surface and velocity expands instantly into smooth undulations. It is therefore necessary to analyse the energy loss across jumps, and juxtapose this analysis between the original and our new extended GreenNaghdi system.

The outline of the paper is as follows. First, a systematic derivation of the new Hamiltonian formulation will be given starting from a slightly adapted formulation of the variational principle of Cotter and Bokhove (2010) in Sect. 2. Subsequently, we show in Sect. 3 how it can be reduced to limiting systems, such as the shallow water equations, the potential flow model, the Boussinesq model of Klopman et al. (2010) and an extended version of the Green-Naghdi equations. In Sect. 5, a variational approach to derive jump conditions is given, starting from the well-known Rankine-Hugoniot or jump conditions for the shallow water equations. We end with conclusions in Sect. 6.

\section{New water wave model}

\subsection{Variational principle}

Consider an incompressible fluid at time $t$ in a threedimensional domain bounded by solid surfaces and a free surface, with horizontal coordinates $x, y$, and vertical coordinate $z$. The water depth is denoted by $h=h(x, y, t)$. There exists a parent Eulerian variational principle for incompressible flow with a free surface. Its three-dimensional velocity field $\boldsymbol{U}=\boldsymbol{U}(x, y, z, t)=(u, v, w)^{T}$, with transpose $(\cdot)^{T}$, contains both potential and rotational parts and is represented as

$\boldsymbol{U}=\nabla \phi+\pi_{j} \nabla \boldsymbol{l}_{j}$

through extended Clebsch variables: the velocity potential $\phi=\phi(x, y, z, t)$, the three-dimensional fluid parcel label $\boldsymbol{l}=$ $\boldsymbol{l}(x, y, z, t)$ and the corresponding Lagrange multiplier vector $\boldsymbol{\pi}=\boldsymbol{\pi}(x, y, z, t)$. Such a representation describes a velocity field containing all three components of vorticity $\boldsymbol{\nabla} \times \boldsymbol{U}$. In order to avoid confusion, indices are also introduced in Eq. (1) and the Einstein convention for repeated indices is used. This velocity representation is similar to the expression (3.9) by Salmon (1988). Also Lin (1963) used two threedimensional vector Clebsch variables to introduce a vorticity for superfluids. As in Yoshida (2009), we see that a pair of extended Clebsch vectors suffices for the generalized form to be complete.

When only the potential velocity field $\nabla \phi(x, y, z, t)$ is used, there is no vorticity. In contrast, a shallow water velocity field includes the vertical component of vorticity. Similarly, in an Eulerian variational principle with planar Clebsch variables that only depend on the horizontal coordinates the vertical component of vorticity is retained. This component is constant throughout the whole water column and flows with helicy (Kuznetsov and Mikhailov, 1980) are thus excluded by construction.

Cotter and Bokhove (2010) derived novel water wave dynamics from the parent Eulerian variational principle which includes two limits: Luke's variational principle giving the classical potential water wave model and a principle for depth-averaged shallow water flows based on planar Clebsch variables. At least conceptually, the novel variational principle follows readily from the parent principle with twodimensional label and multiplier fields $\boldsymbol{l}$ and $\boldsymbol{\pi}$ depending only on the two-dimensional horizontal coordinates and time. Hence, they no longer depend on the vertical coordinate $z$. In his prominent paper, Luke (1967) mentioned about the possibility of the introduction of Clebsch potentials into the variational principle for the rotational case. In contrast, we do not use Clebsch scalar variables, but extended vector Clebsch variables.

Extended Clebsch variables are, however, not convenient to work with. We therefore reduce the model to a more compact and conventional form. This reduction from six 
variables $\{\phi, h, \boldsymbol{l}, \boldsymbol{\pi}\}$ to four more conventional variables $\left\{\phi, h, \boldsymbol{u}^{*}\right\}$ is undertaken in a Hamiltonian setting. The latter variables involve a new velocity $\boldsymbol{u}^{*}$, which is a suitable horizontal velocity.

The variational principle of Cotter and Bokhove (2010) has the following form:

$$
\begin{aligned}
0 & =\delta \int_{0}^{T} \mathcal{L}[\boldsymbol{l}, \boldsymbol{\pi}, \phi, h] \mathrm{d} t \\
& =\delta \int_{0}^{T} \int_{\Omega_{\mathrm{H}}}^{b} \int_{b}^{b+h} \partial_{t} \phi+\pi \cdot \partial_{t} l \mathrm{~d} z \mathrm{~d} x \mathrm{~d} y+\mathcal{H} \mathrm{d} t,
\end{aligned}
$$

where the horizontal part of the domain is $\Omega_{\mathrm{H}}$; the singlevalued free surface boundary lies at $z=h(x, y, t)+b(x, y)$, with $h(x, y, t)$ the water depth and $b(x, y)$ a given, fixed topography; and, $t$ is time, its derivative is $\partial_{t}$, and $T$ a final time. The component of the velocity with vortical parts is contained in

$\mathrm{v}(x, y, t)=\pi_{j}(x, y, t) \nabla l_{j}(x, y, t)$, with $j=1,2$

and three-dimensional gradient $\nabla$. Thus, the entire threedimensional velocity field is represented by

$\boldsymbol{U}(x, y, z, t)=\nabla \phi(x, y, z, t)+\mathbf{v}(x, y, t)$,

combining the potential velocity $\nabla \phi(x, y, z, t)$ and the planar velocity $\mathbf{v}(x, y, t)$. The relevant Hamiltonian, the sum of kinetic and potential energies, equals

$$
\begin{aligned}
\mathcal{H} & =\mathcal{H}[\boldsymbol{l}, \boldsymbol{\pi}, \phi, h]=\int_{\Omega_{\mathrm{H}}} \int_{b}^{b+h} \frac{1}{2}|\nabla \phi+\mathbf{v}|^{2} \mathrm{~d} z \\
& +\frac{1}{2} g\left((h+b)^{2}-b^{2}\right)-g h H_{0} \mathrm{~d} x \mathrm{~d} y,
\end{aligned}
$$

with $g$ the acceleration of the Earth's gravity, and $H_{0}$ a still water reference level. This Hamiltonian is the available potential energy, due to the additional subtraction of the rest level contribution, cf. Shepherd (1993).

As is shown in Cotter and Bokhove (2010), the variational formulation of the new system is similar to Hamiltonian classical mechanics, and becomes

$$
\begin{aligned}
& \delta \phi: \quad \nabla^{2} \phi+\nabla \cdot \mathbf{v}=0, \\
& \delta h: \quad \partial_{t} \phi_{\mathrm{s}}=-\frac{\delta \mathcal{H}}{\delta h}, \quad \delta \phi_{\mathrm{s}}: \quad \partial_{t} h=\frac{\delta \mathcal{H}}{\delta \phi_{\mathrm{s}}}, \\
& \delta(h \boldsymbol{\pi}): \quad \partial_{t} \boldsymbol{l}=-\frac{\delta \mathcal{H}}{\delta(h \boldsymbol{\pi})}, \quad \delta \boldsymbol{l}: \quad \partial_{t}(h \boldsymbol{\pi})=\frac{\delta \mathcal{H}}{\delta \boldsymbol{l}},
\end{aligned}
$$

with Hamiltonian variations equal to

$$
\begin{aligned}
\frac{\delta \mathcal{H}}{\delta h}= & \frac{1}{2}\left|\nabla_{\mathrm{H}} \phi_{\mathrm{S}}+\mathbf{v}\right|^{2}+g\left(h+b-H_{0}\right)-\mathbf{v} \cdot \overline{\boldsymbol{u}} \\
& -\frac{1}{2}\left(\partial_{z} \phi\right)_{\mathrm{S}}^{2}\left(1+\left|\nabla_{\mathrm{H}}(h+b)\right|^{2}\right), \\
\frac{\delta \mathcal{H}}{\delta \phi_{\mathrm{s}}}= & \left(\partial_{z} \phi\right)_{\mathrm{s}}\left(1+\left|\nabla_{\mathrm{H}}(h+b)\right|^{2}\right) \\
& -\left(\nabla_{\mathrm{H}} \phi_{\mathrm{S}}+\mathbf{v}\right) \cdot \nabla_{\mathrm{H}}(h+b), \\
\frac{\delta \mathcal{H}}{\delta\left(h \boldsymbol{\pi}_{i}\right)}= & \overline{\boldsymbol{u}} \cdot \nabla \boldsymbol{l}_{i}, \\
\frac{\delta \mathcal{H}}{\delta \boldsymbol{l}_{i}}= & -\nabla \cdot\left(h \overline{\boldsymbol{u}} \boldsymbol{\pi}_{i}\right) .
\end{aligned}
$$

In the above expressions, we used the depth-averaged horizontal velocity:

$\overline{\boldsymbol{u}}(x, y, t)=\frac{1}{h} \int_{b}^{b+h} \boldsymbol{U}_{\mathrm{H}} \mathrm{d} z$,

where $\boldsymbol{U}_{\mathrm{H}}=(u, v)^{T}$ is the horizontal component of the velocity $\boldsymbol{U}$, and surface velocity potential,

$\phi_{\mathrm{s}}=\phi_{\mathrm{s}}(x, y, t)=\phi(x, y, z=h+b, t)$.

Here the subscript $(.)_{\mathrm{s}}$ denotes evaluation at the free surface.

To obtain these results, we also employed the relation

$\delta\left(\phi_{\mathrm{s}}\right)=(\delta \phi)_{\mathrm{s}}+\left(\partial_{z} \phi\right)_{\mathrm{s}} \delta h$,

and a similar one for $\partial_{t}\left(\phi_{\mathrm{s}}\right)$.

The pairs $(\boldsymbol{l}, h \boldsymbol{\pi})$ and $\left(\phi_{\mathrm{s}}, h\right)$ at the free surface are canonically conjugated. Thus the Hamiltonian dynamics arising from Eqs. (2)-(6) is canonical and takes the form

$\frac{\mathrm{d} \mathcal{F}}{\mathrm{d} t}=\{\mathcal{F}, \mathcal{H}\}$
$\int_{\Omega_{\mathrm{H}}} \frac{\delta \mathcal{F}}{\delta h} \frac{\delta \mathcal{H}}{\delta \phi_{\mathrm{s}}}-\frac{\delta \mathcal{F}}{\delta \phi_{\mathrm{s}}} \frac{\delta \mathcal{H}}{\delta h}+\frac{\delta \mathcal{F}}{\delta(h \boldsymbol{\pi})} \cdot \frac{\delta \mathcal{H}}{\delta \boldsymbol{l}}-\frac{\delta \mathcal{F}}{\delta \boldsymbol{l}} \cdot \frac{\delta \mathcal{H}}{\delta(h \boldsymbol{\pi})} \mathrm{d} x \mathrm{~d} y$.

Subsequent substitution of one of these variables $\boldsymbol{l}, h \boldsymbol{\pi}, \phi_{\mathrm{S}}$ or $h$ - rewritten as a functional in Eq. (11) - in turn yields (6).

\subsection{Reduction of Hamiltonian dynamics}

The aim is to reduce the number of variables in the Hamiltonian formulation from the set $\left\{\phi, \phi_{\mathrm{s}}, h, \boldsymbol{l}, h \boldsymbol{\pi}\right\}$ to the set $\left\{\varphi \equiv \phi-\phi_{\mathrm{s}}, h, \boldsymbol{u}^{*}\right\}$. Doing so removes the reference to the label fields and their conjugates and yields a reduction by two fields. This transformation is achieved via variational techniques. The surface velocity $\boldsymbol{u}^{*}$ is now split into a potential and rotational part, which allows us to reformulate the 
Hamiltonian dynamics. The key observation is that the velocity field (4) can be rewritten as

$\boldsymbol{U}=\boldsymbol{u}^{*}+\nabla\left(\phi-\phi_{\mathrm{s}}\right) \equiv \boldsymbol{u}^{*}+\nabla \varphi$

by introducing a surface velocity

$\boldsymbol{u}^{*}(x, y, t)=\nabla \phi_{\mathrm{s}}(x, y, t)+\mathbf{v}(x, y, t)$.

Upon using this in Eq. (5), the resulting Hamiltonian becomes

$$
\begin{aligned}
\mathcal{H}\left[\phi, \phi_{\mathrm{s}}, h, \boldsymbol{u}^{*}\right]= & \int_{\Omega_{\mathrm{H}}} \int_{b}^{b+h} \frac{1}{2}\left|\boldsymbol{u}^{*}+\nabla\left(\phi-\phi_{\mathrm{s}}\right)\right|^{2} \mathrm{~d} z \\
& +\frac{1}{2} g\left((h+b)^{2}-b^{2}\right) \mathrm{d} x \mathrm{~d} y .
\end{aligned}
$$

Consequently, instead of the seven fields used in Eq. (5), we can use five fields. The question is whether a similar reduction can be achieved from the Poisson bracket, thus closing the Hamiltonian formulation in the new variables. The subsequent derivation has a technical character and readers can safely jump to the next subsection, in which the result is stated.

We relate the two sets of variational derivatives by taking variations of a functional $\mathcal{F}$ in terms of the prognostic variables:

$$
\begin{aligned}
\delta \mathcal{F} & =\int_{\Omega_{\mathrm{H}}} \frac{\delta \mathcal{F}}{\delta \phi_{\mathrm{s}}} \delta \phi_{\mathrm{s}}+\frac{\delta \mathcal{F}}{\delta h} \delta h+\frac{\delta \mathcal{F}}{\delta(h \boldsymbol{\pi})} \cdot \delta(h \boldsymbol{\pi})+\frac{\delta \mathcal{F}}{\delta \boldsymbol{l}} \cdot \delta \boldsymbol{l} \mathrm{d} x \mathrm{~d} y \\
& =\int_{\Omega_{\mathrm{H}}} \frac{\delta \mathcal{F}}{\delta\left(h \boldsymbol{u}^{*}\right)} \cdot \delta\left(h \boldsymbol{u}^{*}\right)+\frac{\delta \mathcal{F}}{\delta h} \delta h \mathrm{~d} x \mathrm{~d} y
\end{aligned}
$$

which connects variations with respect to the different sets of variables. After using (13) with (3) in the above, an integration by parts and using Gauss' law, we obtain

$$
\begin{aligned}
\frac{\delta \mathcal{F}}{\delta \phi_{\mathrm{s}}} & =-\nabla \cdot\left(h \frac{\delta \mathcal{F}}{\delta\left(h \boldsymbol{u}^{*}\right)}\right), \\
\frac{\delta \mathcal{F}}{\delta \boldsymbol{l}_{j}} & =-\nabla \cdot\left(h \boldsymbol{\pi}_{j} \frac{\delta \mathcal{F}}{\delta\left(h \boldsymbol{u}^{*}\right)}\right), \\
\left.\frac{\delta \mathcal{F}}{\delta h}\right|_{\phi_{\mathrm{s}}} & =\frac{\delta \mathcal{F}}{\delta\left(h \boldsymbol{u}^{*}\right)} \cdot \nabla \phi_{\mathrm{s}}+\left.\frac{\delta \mathcal{F}}{\delta h}\right|_{h u^{*}}, \\
\frac{\delta \mathcal{F}}{\delta\left(h \boldsymbol{\pi}_{j}\right)} & =\frac{\delta \mathcal{F}}{\delta\left(h \boldsymbol{u}^{*}\right)} \cdot \nabla \boldsymbol{l}_{j},
\end{aligned}
$$

where also index notation with $i, j, k=1,2$ and $\nabla_{1}=\partial_{x}$, $\nabla_{2}=\partial_{y}$ is used for clarity's sake. Boundary contributions in the above calculation vanish because at solid vertical boundaries $\hat{\boldsymbol{n}}_{\mathrm{H}} \cdot \delta \mathcal{F} / \delta\left(h \boldsymbol{u}^{*}\right)=0$ with the horizontal outward normal $\hat{\boldsymbol{n}}_{\mathrm{H}}$, or because $h=0$ at the water line. Substitution of (16) into (11) yields the transformed Hamiltonian formulation in momentum variables:

$$
\begin{aligned}
& \frac{\mathrm{d} \mathcal{F}}{\mathrm{d} t}=\int_{\Omega_{\mathrm{H}}} \frac{\delta \mathcal{H}}{\delta h} \nabla_{i}\left(h \frac{\delta \mathcal{F}}{\delta\left(h \boldsymbol{u}_{i}^{*}\right)}\right)-\frac{\delta \mathcal{F}}{\delta h} \nabla_{i}\left(h \frac{\delta \mathcal{H}}{\delta\left(h \boldsymbol{u}_{i}^{*}\right)}\right) \\
& +h \boldsymbol{u}_{k}^{*}\left(\frac{\delta \mathcal{H}}{\delta\left(h \boldsymbol{u}_{k}^{*}\right)} \nabla_{i} \frac{\delta \mathcal{F}}{\delta\left(h \boldsymbol{u}_{i}^{*}\right)}-\frac{\delta \mathcal{F}}{\delta\left(h \boldsymbol{u}_{k}^{*}\right)} \nabla_{i} \frac{\delta \mathcal{H}}{\delta\left(h \boldsymbol{u}_{i}^{*}\right)}\right) \\
& +\frac{\delta \mathcal{F}}{\delta\left(h \boldsymbol{u}_{i}^{*}\right)} \frac{\delta \mathcal{H}}{\delta\left(h \boldsymbol{u}_{k}^{*}\right)}\left(\nabla_{i}\left(h \boldsymbol{u}_{k}^{*}\right)-\nabla_{k}\left(h \boldsymbol{u}_{i}^{*}\right)\right) \mathrm{d} x \mathrm{~d} y,
\end{aligned}
$$

where we employed the chain rule, the relation

$\nabla_{i} h \nabla_{k} \phi_{\mathrm{S}}-\nabla_{k} h \nabla_{i} \phi_{\mathrm{S}}=\nabla_{i}\left(h \nabla_{k} \phi_{\mathrm{S}}\right)-\nabla_{k}\left(h \nabla_{i} \phi_{\mathrm{s}}\right)$

and $\nabla_{i} \nabla_{k} \phi_{\mathrm{s}}=\nabla_{k} \nabla_{i} \phi_{\mathrm{s}}$.

\subsection{Hamiltonian dynamics of new water wave model}

We complete the derivation by stating the Hamiltonian dynamics of the new water wave model. In the next two sections, two limiting systems and Boussinesq approximations will be based directly on this new Hamiltonian formulation. The final step is to transform the Hamiltonian formulation (17) with respect to the set $\left\{h, h \boldsymbol{u}^{*}\right\}$ into one with respect to $\left\{h, \boldsymbol{u}^{*}\right\}$, using the relations

$\frac{\delta \mathcal{F}}{\delta\left(h \boldsymbol{u}^{*}\right)}=\frac{1}{h} \frac{\delta \mathcal{F}}{\delta \boldsymbol{u}^{*}} \quad$ and $\left.\quad \frac{\delta \mathcal{F}}{\delta h}\right|_{h u^{*}}=\left.\frac{\delta \mathcal{F}}{\delta h}\right|_{u^{*}}-\frac{\boldsymbol{u}^{*}}{h} \frac{\delta \mathcal{F}}{\delta \boldsymbol{u}^{*}}$.

By substitution of (19) into (17), we obtain the desired Hamiltonian formulation in the new variables:

$$
\begin{aligned}
\frac{\mathrm{d} \mathcal{F}}{\mathrm{d} t}= & \int_{\Omega_{\mathrm{H}}}-q \frac{\delta \mathcal{F}}{\delta \boldsymbol{u}^{*}} \cdot \frac{\delta \mathcal{H}^{\perp}}{\delta \boldsymbol{u}^{*}} \\
& -\frac{\delta \mathcal{F}}{\delta h} \nabla \cdot \frac{\delta \mathcal{H}}{\delta \boldsymbol{u}^{*}}+\frac{\delta \mathcal{H}}{\delta h} \nabla \cdot \frac{\delta \mathcal{F}}{\delta \boldsymbol{u}^{*}} \mathrm{~d} x \mathrm{~d} y,
\end{aligned}
$$

with $(\cdot)^{\perp}$ the rotated vector as in $\boldsymbol{u}^{* \perp} \equiv\left(-u_{2}^{*}, u_{1}^{*}\right)^{T}$, and note that the gradients $\nabla$ are effectively two dimensional as they operate on functions independent of $z$. The potential vorticity is defined as

$$
\begin{aligned}
q \equiv\left(\partial_{x} v-\partial_{y} u\right) / h & =\left(\partial_{x} \mathrm{v}_{2}-\partial_{y} \mathrm{v}_{1}\right) / h \\
& =\left(\partial_{x} u_{2}^{*}-\partial_{y} u_{1}^{*}\right) / h .
\end{aligned}
$$

No integration by parts was required in the previous transformation. Since only the difference of variables $\phi$ and $\phi_{\mathrm{S}}$ appears, we introduce a modified potential $\varphi=\phi-\phi_{\mathrm{s}}$, zero at the free surface. Hence, we can slightly simplify Eq. (14) to

$$
\begin{aligned}
\mathcal{H}\left[\varphi, h, \boldsymbol{u}^{*}\right]=\int_{\Omega_{\mathrm{H}}} \int_{b}^{b+h} \frac{1}{2}\left|\boldsymbol{u}^{*}+\nabla \varphi\right|^{2} \mathrm{~d} z & \\
& \quad+\frac{1}{2} g\left((h+b)^{2}-b^{2}\right) \mathrm{d} x \mathrm{~d} y .
\end{aligned}
$$


Specification of $\mathcal{F}$ in (20), in turn, and use of (22), yields the equations of motion:

$$
\begin{aligned}
\partial_{t} h & =-\nabla \cdot \frac{\delta \mathcal{H}}{\delta \boldsymbol{u}^{*}}, \\
\partial_{t} \boldsymbol{u}^{*} & =-\nabla \frac{\delta \mathcal{H}}{\delta h}-q \frac{\delta \mathcal{H}}{\delta \boldsymbol{u}^{*}},
\end{aligned}
$$

using Hamiltonian variations:

$\delta h: \frac{\delta \mathcal{H}}{\delta h}=B$,

$\delta \boldsymbol{u}^{*}: \quad \frac{\delta \mathcal{H}}{\delta \boldsymbol{u}^{*}}=h \overline{\boldsymbol{u}}$,

$\delta \varphi: \quad-\frac{\delta \mathcal{H}}{\delta \varphi}=\nabla \cdot \boldsymbol{u}^{*}+\nabla^{2} \varphi=0$,

with the depth-weighted horizontal velocity vector in (8) redefined as

$\overline{\boldsymbol{u}}(x, y, t)=\frac{1}{h} \int_{b}^{b+h}\left(\boldsymbol{u}^{*}+\nabla_{\mathrm{H}} \varphi\right) \mathrm{d} z$,

and the Bernoulli function:

$B=\frac{1}{2}\left|\boldsymbol{u}^{*}\right|^{2}+g(h+b)-\frac{1}{2}\left(\partial_{z} \varphi\right)_{\mathrm{s}}^{2}\left(1+\left|\nabla_{\mathrm{H}}(h+b)\right|^{2}\right)$.

Note that $\delta \mathcal{H} / \delta \varphi=0$ acts here as a constraint, since it does not play a role in the prognostics.

The final system of equations in the new free surface variables equals

$$
\begin{aligned}
\partial_{t} h+\nabla \cdot(h \overline{\boldsymbol{u}}) & =0, \\
\partial_{t} \boldsymbol{u}^{*}+\nabla B+q(h \overline{\boldsymbol{u}})^{\perp} & =0,
\end{aligned}
$$

with the elliptic equation for $\varphi$ in the interior:

$\nabla^{2} \varphi=-\nabla \cdot u^{*}$.

The boundary conditions for $\varphi$ in (28) are $\boldsymbol{n} \cdot\left(\boldsymbol{u}^{*}+\nabla \varphi\right)=0$ at solid walls, with $\boldsymbol{n}$ the exterior normal vector, and $\varphi=0$ at the free surface.

We can also formulate the new system in a (conservative) form, which will become relevant for the derivation of jump conditions later. Using definitions (25) and (12), the key step is to notice that

$$
\begin{aligned}
\partial_{t}(h \overline{\boldsymbol{u}}) & =\partial_{t}\left(\int_{b}^{b+h} \boldsymbol{U}_{\mathrm{H}} \mathrm{d} z\right) \\
& =\left(u_{\mathrm{s}}, v_{\mathrm{s}}\right)^{T} \partial_{t} h+h \partial_{t} \boldsymbol{u}^{*}+\int_{b}^{b+h} \nabla_{\mathrm{H}} \partial_{t} \varphi \mathrm{d} z .
\end{aligned}
$$

The term $h \partial_{t} \boldsymbol{u}^{*}$ can now be obtained from (27b). The integral term is rewritten by interchanging the order of integral and horizontal gradients, thus introducing surface and bottom boundary contributions. The next step is to rewrite the continuity equation (24c), or $\boldsymbol{\nabla} \cdot \boldsymbol{U}=0$, by integrating over depth, to obtain

$\partial_{t} h=w_{\mathrm{s}}-u_{\mathrm{s}} \partial_{x}(b+h)-v_{\mathrm{s}} \partial_{y}(b+h)$,

in which we use the full velocity evaluated at the free surface and we note that $w_{\mathrm{s}}=\left(\partial_{z} \varphi\right)_{\mathrm{s}}$. Hence, we can evaluate each term in (29) further. Substitution of (30) into (29) leads to terms like $-u_{\mathrm{s}}^{2} \partial_{x}(b+h)-u_{\mathrm{s}} v_{\mathrm{s}} \partial_{y}(b+h)$, which can be rewritten in terms of depth-integrated fluxes of the threedimensional velocity. For example, $u_{\mathrm{s}}^{2} \partial_{x}(b+h)$ can be determined from

$\partial_{x} \int_{b}^{b+h} u^{2} \mathrm{~d} z=\int_{b}^{b+h} 2 u \partial_{x} u \mathrm{~d} z+u_{\mathrm{s}}^{2} \partial_{x}(b+h)-u_{\mathrm{b}}^{2} \partial_{x} b$

in which subscript $(\cdot)_{\mathrm{b}}$ in $u_{\mathrm{b}}$ denotes that horizontal velocity $u$ is evaluated at the bottom $z=b$. For gradients at the free surface, we extensively use relations like

$(\nabla \varphi)_{\mathrm{s}}=\left(\nabla \varphi_{\mathrm{s}}\right)-\left(\partial_{z} \phi\right)_{\mathrm{s}} \nabla(h+b)=-w_{\mathrm{s}} \nabla(h+b)$,

since by definition $\varphi_{\mathrm{s}}=0$. In addition, we use the condition that the velocity normal to the bottom boundary is zero.

Without going through further details, the reformulated equations of motion resulting after some calculations become as follows:

$\partial_{t}\left(\begin{array}{c}h \\ h \bar{u} \\ h \bar{v}\end{array}\right)+\nabla \cdot\left(\begin{array}{c}\mathcal{F}_{0} \\ \mathcal{F}_{1} \\ \mathcal{F}_{2}\end{array}\right)=\left(\begin{array}{c}0 \\ S_{1} \\ S_{2}\end{array}\right)$,

with the flux tensor

$$
\left(\begin{array}{l}
\mathcal{F}_{0} \\
\mathcal{F}_{1} \\
\mathcal{F}_{2}
\end{array}\right)=\left(\begin{array}{cc}
h \bar{u} & h \bar{v} \\
A & \int_{b}^{h+b} u v \mathrm{~d} z \\
\int_{b}^{h+b} u v \mathrm{~d} z & C
\end{array}\right),
$$

where $\overline{\boldsymbol{u}}=(\bar{u}, \bar{v})^{T}$,

$$
\begin{aligned}
A= & \int_{b}^{h+b}\left(u^{2}-\frac{|\boldsymbol{U}|^{2}}{2}+\frac{\left|\boldsymbol{u}^{*}\right|^{2}}{2}-\partial_{t} \varphi\right) \mathrm{d} z+\frac{g h^{2}}{2} \\
& -\frac{h}{2} w_{\mathrm{s}}^{2}\left(1+|\nabla(h+b)|^{2}\right), \\
C= & \int_{b}^{h+b}\left(v^{2}-\frac{|\boldsymbol{U}|^{2}}{2}+\frac{\left|\boldsymbol{u}^{*}\right|^{2}}{2}-\partial_{t} \varphi\right) \mathrm{d} z+\frac{g h^{2}}{2} \\
& -\frac{h}{2} w_{\mathrm{s}}^{2}\left(1+|\nabla(h+b)|^{2}\right),
\end{aligned}
$$

and

$$
\begin{aligned}
\left(S_{1}, S_{2}\right)^{T}= & \left(-g h+\frac{1}{2} w_{\mathrm{s}}^{2}\left(1+|\nabla(h+b)|^{2}\right)\right. \\
& \left.+\left(\partial_{t} \varphi+\frac{1}{2}|\boldsymbol{U}|^{2}-\frac{1}{2}\left|\boldsymbol{u}^{*}\right|^{2}\right)_{b}\right)\left(\partial_{x} b, \partial_{y} b\right)^{T} .
\end{aligned}
$$




\section{Shallow water and potential flow limits}

The shallow water and potential flow models emerge as limiting systems of the new water wave model, as will be shown next. The new water wave model reduces to the potential flow equations when we take $\boldsymbol{U}=\boldsymbol{\nabla} \phi$ in the Hamiltonian (5) and only use the terms with $h$ and $\phi_{\mathrm{S}}$ in the Poisson bracket (11). The Hamiltonian of the system then takes the form

$\mathcal{H}=\mathcal{H}[\phi, h]=$

$\int_{\Omega_{\mathrm{H}}} \int_{b}^{b+h} \frac{1}{2}|\nabla \phi|^{2} \mathrm{~d} z+\frac{1}{2} g\left((h+b)^{2}-b^{2}\right)-g h H_{0} \mathrm{~d} x \mathrm{~d} y$.

The shallow water limit is obtained when we restrict $\phi=$ $\phi(x, y, z, t)$ to be the surface potential $\phi_{\mathrm{S}}=\phi_{\mathrm{S}}(x, y, t)$ in the extended Luke's variational principle (2) such that $\varphi=0$. The velocity field then reduces to $\boldsymbol{U}(x, y, t)=\boldsymbol{u}^{*}(x, y, t)=$ $\nabla \phi_{\mathrm{S}}(x, y, t)+\mathbf{v}(x, y, t)$. This change yields $\overline{\boldsymbol{u}}=\boldsymbol{u}^{*}(x, y, t)$, and the Hamiltonian dynamics remains (20) but with the Hamiltonian:

$\mathcal{H}=\mathcal{H}[\overline{\boldsymbol{u}}, h]=$

$\int_{\Omega_{\mathrm{H}}} \frac{1}{2} h|\overline{\boldsymbol{u}}|^{2}+\frac{1}{2} g\left((h+b)^{2}-b^{2}\right) \mathrm{d} x \mathrm{~d} y$,

cf. (Salmon, 1988). In this case Eq. (27b) is transformed to the depth-averaged shallow water momentum equation:

$\partial_{t} \overline{\boldsymbol{u}}+\nabla B+q h \overline{\boldsymbol{u}}^{\perp}=0$,

with $q h=\partial_{x} \bar{v}-\partial_{y} \bar{u}$ and $B=(1 / 2)|\overline{\boldsymbol{u}}|^{2}+g(h+b)$.

\section{Hamiltonian Boussinesq reductions of new model}

The idea to approximate the vertical structure of the flow velocity beneath the free surface was first applied by Boussinesq (1871) for the description of fairly long surface waves in shallow water. Such Boussinesq-type water wave models are widely used in coastal and maritime engineering. Alternatively, these models can be viewed as a Galerkin or Ritz discretization of the velocity potential in the vertical coordinate $z$ only. When such an expansion of the velocity potential in terms of vertical profiles is substituted directly into the variational principle, a so-called variational Boussinesq model results. It depends on only the horizontal coordinates and time. An example is the variational Boussinesq model of Klopman et al. (2010). These authors also sketched how to add a vorticity term to the potential flow model, but in an ad hoc fashion. In contrast, we apply the Galerkin or Ritz method directly to the Hamiltonian formulation of our new water wave model, and thus systematically maintain the vertical component of the vorticity. A Boussinesq-type wave model can subsequently also be discretized in the horizontal directions and time. It is unclear whether such a secondary discretization instead of one directly applied to the original model in three dimensions is more advantageous, or not. The advantage of first discretizing the vertical direction may be that these reduced Boussinesq models are more amenable to mathematical analysis. The analysis of jump conditions, explored later, perhaps illustrates this point.

\subsection{Variational Boussinesq model}

In the Ritz method, the velocity potential is approximated as a linear combination of $M$ basis functions, such that

$\varphi(x, y, z, t)=\sum_{m=1}^{M} f_{m}\left(z ; h, b, k_{m}\right) \psi_{m}(x, y, t)$,

with shape functions $f_{m}$ and variables $\psi_{m}(x, y, t)$. By definition, the shape functions are chosen such that $f_{m}=0$ at the free surface $z=h+b$ in a strong sense. The functions $k_{m}(x, y)$ may be used as optional shape parameters, but we assume them to be known and fixed a priori. Note that due to the direct substitution of (40) into Eq. (22), the Hamiltonian remains by default positive. The expansion (40) implies that the condition $\delta \mathcal{H} / \delta \varphi=0$ is replaced by

$$
\frac{\delta \mathcal{H}}{\delta \psi_{m}}=0, \quad m=1, \ldots, M .
$$

The simplest model of practical interest has one shape function $(M=1)$ :

$\varphi(x, y, z, t)=f(z ; b, h) \psi(x, y, t)$,

and the following expression for the flow velocity is obtained:

$$
\begin{aligned}
\nabla_{\mathrm{H}} \varphi & =f \nabla_{\mathrm{H}} \psi+\left(\partial_{b} f\right) \psi \nabla_{\mathrm{H}} b+\left(\partial_{h} f\right) \psi \nabla_{\mathrm{H}} h, \\
\partial_{z} \phi & =\left(\partial_{z} f\right) \psi .
\end{aligned}
$$

In principle it seems that a substitution of Eq. (43) into the Hamiltonian (22) combined with the Hamiltonian dynamics (20) suffices to define a reduced Boussinesq model. The challenge, however, is to satisfy the bottom boundary condition:

$w=\psi \partial_{z} f=\left(\boldsymbol{u}^{*}+\nabla_{\mathrm{H}}(f \psi)\right) \cdot \nabla_{\mathrm{H}} b \quad$ at $\quad z=b$

in a strong sense. Satisfaction of this bottom boundary condition in a weak sense, as in numerical approaches, appears to be less well explored (in Boussinesq water wave models).

It is therefore common (cf. Klopman et al., 2010) to assume the bed slopes to be mild, such that $\nabla_{\mathrm{H}} b \approx 0$ and (43) can be approximated as

$$
\begin{aligned}
\nabla_{\mathrm{H}} \varphi & =f \nabla_{\mathrm{H}} \psi+\left(\partial_{h} f\right) \psi \nabla_{\mathrm{H}} h, \\
\partial_{z} \phi & =\left(\partial_{z} f\right) \psi .
\end{aligned}
$$


Consequently, (44) reduces to $\partial_{z} f=0$, which is more easily imposed on the vertical profile $f(z ; b, h)$ in a strong sense. After introducing (45) into the Hamiltonian (22), the result is

$$
\begin{aligned}
\mathcal{H}= & \int_{\Omega_{\mathrm{H}}} \int_{b}^{h+b} \frac{1}{2}\left|\boldsymbol{u}^{*}+f \nabla_{\mathrm{H}} \psi+\left(\partial_{h} f\right) \psi \nabla_{\mathrm{H}} h\right|^{2} \\
& +\frac{1}{2}\left(\psi \partial_{z} f\right)^{2} \mathrm{~d} z+\frac{1}{2} g\left((h+b)^{2}-b^{2}\right) \mathrm{d} x \mathrm{~d} y \\
= & \int_{\Omega_{\mathrm{H}}} \frac{1}{2} h\left|\boldsymbol{u}^{*}\right|^{2}+\frac{1}{2} F|\nabla \psi|^{2}+P \nabla \psi \cdot \boldsymbol{u}^{*} \\
& +\frac{1}{2} \psi^{2}\left(K+G|\nabla h|^{2}\right)+Q \psi \boldsymbol{u}^{*} \cdot \nabla h+R \psi \nabla \psi \cdot \nabla h \\
& +\frac{1}{2} g\left((h+b)^{2}-b^{2}\right) \mathrm{d} x \mathrm{~d} y,
\end{aligned}
$$

where $F, K, G, P, Q, R$ are functions of $h$, provided in Appendix A. Variations of (46) with respect to $h, \boldsymbol{u}^{*}$ remain as in (24a) and (24b), but the elliptic equation (24c), here resulting from the variation of $\psi$, is reduced to

$$
\begin{gathered}
\delta \psi: \quad\left(K+G|\nabla h|^{2}\right) \psi+Q \boldsymbol{u}^{*} \cdot \nabla h+R \nabla \psi \cdot \nabla h \\
-\nabla \cdot\left(F \nabla \psi+P \boldsymbol{u}^{*}+R \psi \nabla h\right)=0 .
\end{gathered}
$$

Perhaps, it is a matter of taste whether (47) is simpler than (28). The reduction in dimensionality, however, is clear, as (28) is an elliptic equation in a three-dimensional domain, while (47) holds in the corresponding horizontal domain defined by the (single-valued) free surface. These variations, combined with Hamiltonian dynamics (20), again yield the system (23). The expressions for the depth-averaged horizontal velocity and the Bernoulli function are, however, modified as follows:

$$
\begin{aligned}
h \overline{\boldsymbol{u}} & =\int_{b}^{b+h}\left(\boldsymbol{u}^{*}+f \nabla_{\mathrm{H}} \psi+\left(\partial_{h} f\right) \psi \nabla_{\mathrm{H}} h\right) \mathrm{d} z \\
& =h \boldsymbol{u}^{*}+P \nabla \psi+Q \psi \nabla h, \\
B & =\frac{1}{2}\left|\boldsymbol{u}^{*}\right|^{2}+g(h+b)+\mathcal{R}^{*},
\end{aligned}
$$

with $\mathcal{R}^{*}$ defined as

$$
\begin{aligned}
\mathcal{R}^{*}= & \frac{1}{2} F^{\prime}|\nabla \psi|^{2}+\frac{1}{2}\left(K^{\prime}+G^{\prime}|\nabla h|^{2}\right) \psi^{2} \\
& +\left(P^{\prime} \nabla \psi+Q^{\prime} \psi \nabla h\right) \cdot \boldsymbol{u}^{*}+R^{\prime} \psi \nabla \psi \cdot \nabla h \\
& -\nabla \cdot\left(G \psi^{2} \nabla h+Q \psi \boldsymbol{u}^{*}+R \psi \nabla \psi\right),
\end{aligned}
$$

and primed variables denote $P^{\prime}=\mathrm{d} P / \mathrm{d} h$, etc. In the variations of (46) with respect to $\psi$ and $h$, boundary contributions cancel either because the velocity normal to vertical walls is zero or because $h=0$ at the water line. Note that the approximated system of equations again takes the form (27) augmented with the elliptic equation (47) for $\psi$.

When, for example, we consider a parabolic vertical profile

$$
f=f^{(p)}=\frac{1}{2} \frac{(z-b)^{2}-h^{2}}{h},
$$

then the Hamiltonian becomes

$$
\begin{aligned}
\mathcal{H}= & \int_{\Omega_{\mathrm{H}}} \frac{1}{2} h\left|\boldsymbol{u}^{*}-\frac{2}{3} \psi \nabla h-\frac{1}{3} h \nabla \psi\right|^{2}+\frac{1}{2} g\left((h+b)^{2}-b^{2}\right) \\
& +\frac{1}{90} h|\psi \nabla h-h \nabla \psi|^{2}+\frac{1}{6} h \psi^{2} \mathrm{~d} x \mathrm{~d} y,
\end{aligned}
$$

which is positive-definite, since the water depth $h>0$. The integrals $F, K, G, P, Q$, and $R$ are readily calculated explicitly, see Appendix A. Consequently, one finds that the relevant expressions become

$$
\begin{gathered}
h \overline{\boldsymbol{u}}=h \boldsymbol{u}^{*}-\frac{1}{3} h^{2} \nabla \psi-\frac{2}{3} h \psi \nabla h, \\
B=\frac{1}{2}\left|\boldsymbol{u}^{*}\right|^{2}+g(h+b)+\mathcal{R}^{*}, \\
\mathcal{R}^{*}=\frac{1}{5} h^{2}|\nabla \psi|^{2}+\frac{1}{6}\left(1+\frac{7}{5}|\nabla h|^{2}\right) \psi^{2} \\
\quad-\frac{2}{3}(h \nabla \psi+\psi \nabla h) \cdot \boldsymbol{u}^{*}+\frac{2}{5} h \psi \nabla \cdot \psi \nabla h \\
\quad-\nabla \cdot\left(\frac{7}{15} h \psi^{2} \nabla h-\frac{2}{3} h \psi \boldsymbol{u}^{*}+\frac{1}{5} h^{2} \psi \nabla \psi\right), \\
h \psi\left(\frac{1}{3}+\frac{7}{15}|\nabla h|^{2}\right)-\left(\frac{2}{3} h \boldsymbol{u}^{*}-\frac{1}{5} h^{2} \nabla \psi\right) \cdot \nabla h \\
\quad-\nabla \cdot\left(\frac{2}{15} h^{3} \nabla \psi-\frac{1}{3} h^{2} \boldsymbol{u}^{*}+\frac{1}{5} h^{2} \psi \nabla h\right)=0 .
\end{gathered}
$$

In summary, we derived and extended the variational Boussinesq model within a Hamiltonian framework, by a Ritz and mild-slope approximation of the vertical potential flow profile, while systematically including the vertical component of the vorticity. The difference between our model and Klopman's model is in the velocity field, which in our case includes the vertical vorticity. The surface velocity representation $\boldsymbol{u}^{*}(x, y, t)=\nabla \phi_{\mathrm{S}}(x, y, t)+\mathbf{v}(x, y, t)$ namely replaces the representation used by Klopman $\boldsymbol{u}^{*}(x, y, t)=$ $\nabla \phi_{\mathrm{s}}(x, y, t)$ from the onset.

\subsection{Green-Naghdi limit}

The Green-Naghdi equations are obtained from a variational principle under the assumption that the fluid moves in vertical columns, as was shown by Miles and Salmon (1985). The 
model is sufficiently dispersive that shocks cannot be maintained as an initial discontinuity disperses into smooth undulations instantly, as was shown by El et al. (2006). We will show that the Green-Naghdi equations can be derived from the variational Boussinesq model with a parabolic potential flow profile via an additional approximation to the Hamiltonian.

Instead of (51), the shape function is taken to be $\left(h^{2}-(z-b)^{2}\right) / 2$. Hence, the modified velocity potential becomes

$\varphi(x, y, z, t)=\frac{h^{2}-(z-b)^{2}}{2} \psi(x, y, t)$.

Of course, this is equivalent to (42) with (51), i.e. $\varphi^{(p)}=$ $\left((z-b)^{2}-h^{2}\right) \psi^{(p)} /(2 h)$, provided we redefine $\psi^{(p)}=$ $-h \psi$. With the mild-slope approximation, the velocity field then becomes

$\boldsymbol{u}_{\mathrm{H}}=\boldsymbol{u}^{*}+\frac{1}{2} \nabla_{\mathrm{H}}\left(\left(h^{2}-z^{2}\right) \psi\right)$,

$w=\varphi_{z}=-z \psi$.

The expressions (52) and (53) are now immediately valid given this substitution of $\psi^{(p)}$ in terms of $h$ and $\psi$. The depth-averaged velocity thus follows from (53a) as

$\overline{\boldsymbol{u}}=\frac{1}{h} \int_{b}^{h+b} \boldsymbol{u} \mathrm{d} z=\boldsymbol{u}^{*}+h \psi \nabla h+\frac{h^{2}}{3} \nabla \psi$.

Likewise, the Hamiltonian (52) becomes

$$
\begin{gathered}
\mathcal{H}\left[h, \boldsymbol{u}^{*}, \psi\right]=\int_{\Omega} \frac{1}{2} h|\overline{\boldsymbol{u}}|^{2}+\frac{1}{6} h^{3} \psi^{2}+\frac{1}{2} g\left((h+b)^{2}-b^{2}\right) \\
+\beta \frac{h^{5}|\nabla \psi|^{2}}{90} \mathrm{~d} x \mathrm{~d} y,
\end{gathered}
$$

where we added a "switch" parameter $\beta=\{0,1\}$ to be used later, and rephrased the formulation in terms of $\overline{\boldsymbol{u}}$. Note, however, that $\overline{\boldsymbol{u}}$ is defined in terms of $h, \psi$ and $\boldsymbol{u}^{*}$ in (56).

The Hamiltonian dynamics (23) combined with variations of (57) with respect to $h$ and $\boldsymbol{u}^{*}$ (using Eq. 56) again lead to the dynamics (27). Either via (47) or more directly by taking variations with respect to $\psi$ for fixed $h$ and $\boldsymbol{u}^{*}$ in (57), one obtains

$\psi=\nabla \cdot \overline{\boldsymbol{u}}+\frac{\beta}{15 h^{3}} \nabla \cdot\left(h^{5} \nabla \psi\right)$.

This is an elliptic equation for $\psi$ once one uses (56) to reexpress $\overline{\boldsymbol{u}}$. The Bernoulli function follows either by rearranging (49) or from the variation of (57) with respect to $h$, and takes the form

$$
\begin{aligned}
B=\frac{1}{2}|\overline{\boldsymbol{u}}|^{2} & +\frac{1}{2} h^{2} \psi^{2}+g(h+b)-\frac{1}{3} h^{2} \overline{\boldsymbol{u}} \cdot \nabla \psi \\
& -h^{2} \psi \nabla \cdot \overline{\boldsymbol{u}}-h \psi \overline{\boldsymbol{u}} \cdot \nabla h+\beta h^{2} \frac{h^{2}|\nabla \psi|^{2}}{18} .
\end{aligned}
$$

The Green-Naghdi system arises by keeping the relation (56) between $\overline{\boldsymbol{u}}$ and $\boldsymbol{u}^{*}$ and the Hamiltonian dynamics (23), but simplifying the Hamiltonian (57) to one with $\beta=0$. Hence, the variations with respect to $h$ and $\psi$ and the equations (58) simplify to

$$
\begin{aligned}
& \psi=\nabla \cdot \overline{\boldsymbol{u}}, \\
& B=\frac{1}{2}|\overline{\boldsymbol{u}}|^{2}-\frac{1}{2} h^{2}(\nabla \cdot \overline{\boldsymbol{u}})^{2}+g(h+b)-\frac{1}{3} h^{2} \overline{\boldsymbol{u}} \cdot \nabla(\nabla \cdot \overline{\boldsymbol{u}}) \\
& -(h \boldsymbol{\nabla} \cdot \overline{\boldsymbol{u}})(\overline{\boldsymbol{u}} \cdot \nabla h) .
\end{aligned}
$$

This simplification of the Hamiltonian is equivalent to the substitution of yet another three-dimensional velocity

$\boldsymbol{u}=\tilde{\boldsymbol{u}}=(\overline{\boldsymbol{u}},-z \psi)^{T}$

into the original Hamiltonian (22). Consequently, (59a) is a continuity equation given a columnar horizontal velocity $\overline{\boldsymbol{u}}$ and that $w=-z \psi$. Due to this approximation, the velocity field given by (60) has nonzero horizontal vorticity components:

$\boldsymbol{\omega}=\nabla \times(\bar{u}, \bar{v}, w)^{T}=\left(\partial_{y} w,-\partial_{x} w, \partial_{x} \bar{v}-\partial_{y} \bar{u}\right)$,

in contrast to the original system with $\beta=1$.

The explicit expression $\psi=\nabla \cdot \overline{\boldsymbol{u}}$ in Eq. (59a) allows us to reformulate the system to the standard Green-Naghdi model, as follows:

$$
\begin{aligned}
\partial_{t} h & +\nabla \cdot(h \overline{\boldsymbol{u}})=0, \\
\partial_{t} \overline{\boldsymbol{u}} & +(\overline{\boldsymbol{u}} \cdot \nabla) \overline{\boldsymbol{u}}+g \nabla(h+b) \\
& =h \nabla h\left(\nabla \cdot \partial_{t} \overline{\boldsymbol{u}}+(\overline{\boldsymbol{u}} \cdot \nabla)(\nabla \cdot \overline{\boldsymbol{u}})-(\nabla \cdot \overline{\boldsymbol{u}})^{2}\right) \\
& +\frac{h^{2}}{3} \nabla\left(\nabla \cdot \partial_{t} \overline{\boldsymbol{u}}+(\overline{\boldsymbol{u}} \cdot \nabla)(\nabla \cdot \overline{\boldsymbol{u}})-(\nabla \cdot \overline{\boldsymbol{u}})^{2}\right),
\end{aligned}
$$

cf. Eq. (1) in Bonneton et al. (2010). In summary, we have recovered the original Green-Naghdi system from a reformulation and approximation of the variational Boussinesq model. This approximation is Hamiltonian, but consists of using another, columnar approximation of the three-dimensional velocity in the Hamiltonian rather than employing the parabolic potential profile that is still used in the Poisson bracket.

\section{Jump conditions for bores}

The most widely used model to describe wave propagation and breaking near the shore - the shallow water equations does not contain dispersion. Nevertheless, dispersive effects during wave propagation in coastal zones can be important. We illustrate the subtle interplay between dispersion and dissipation with the bore-soliton-splash experiment (Bokhove et al., 2011). This experiment is conducted in a wave channel with a sluice at the beginning and a constriction at the end. The sluice gate locks in a higher water level than in the 
main part of the channel. At some point this gate is opened instantly and a soliton is formed (see Fig. 1), which breaks quickly because its amplitude is too high and propagates further as a hydraulic jump or bore (see Fig. 2). During its propagation the bore loses energy and amplitude, such that just before the constriction, it turns into the smooth soliton again (see Fig. 3). The first reflected soliton draws a through at the contraction in which the lower second soliton crashes and splashes up (see Fig. 4). We mention that there were three "nearly" similar reruns of the experiment, and we used the best images from any of these three (Zweers, 2010). The discussion concerns runs 3,6 , and 8 (performed at the opening of the education plaza at the University of Twente in 2010). The propagation of a smooth, broken and rejuvenated soliton is an illustration of the balance and imbalance between nonlinearity and dispersion. Therefore, a theoretical and numerical model to describe such a phenomena has to include dispersion and has to deal with breaking waves, in which nonlinearity dominates.

Following ideas of Wakelin (1993), we further develop a technique to derive jump conditions from variational principles. To illustrate the intricate details of this approach, the well-known jump conditions for bores are derived first for the depth-averaged shallow water equations in one dimension. Subsequently, the jump conditions for the new water wave model in two horizontal dimensions are obtained and its limitation to the well-known 2-D shallow water jump conditions is shown. The jump conditions for the closely related variational Boussinesq and the Green-Naghdi models are especially interesting as far as it is known that the GreenNaghdi model cannot maintain discontinuities since dispersion is too strong (El et al., 2006). The situation for the variational Boussinesq model is unknown, while we know that the full water wave model with its potential flow water waves can lead to overturning and breaking waves.

\subsection{1-D jump conditions for shallow water equations}

Consider a bore propagating in a channel $\Omega$. The domain $\Omega$ is split into two parts: $\Omega_{1}$ lying behind the bore and $\Omega_{2}$ lying in front of the bore, as shown in Fig. 5. Between these domains there is a vertical moving boundary $\partial \Omega_{\mathrm{b}}$ corresponding to the instant bore position at $x=x_{\mathrm{b}}(t)$. The key point is to consider the two domains separately and couple them at $x_{\mathrm{b}}$. If we consider one subdomain, then the moving bore interface is akin to a piston wave maker. It will be shown that variational techniques are a natural way to obtain the bore relations. The coupling establishes that there is an energy loss at the interior bore boundary.

Let us assume that the domain $\Omega$ has solid wall boundaries and a flat bottom. The state to the left from the interior bore boundary $x_{\mathrm{b}}(t)$ is given by the depth $h^{-}$and horizontal velocity $u^{-}$, and the one to the right by $h^{+}$and $u^{+}$. The bore speed $S=\dot{x}_{\mathrm{b}} \equiv \mathrm{d} x_{\mathrm{b}} / \mathrm{d} t$. The shallow water velocity potential considered at the free surface is $\phi \equiv \phi_{\mathrm{s}}(x, t)$, with

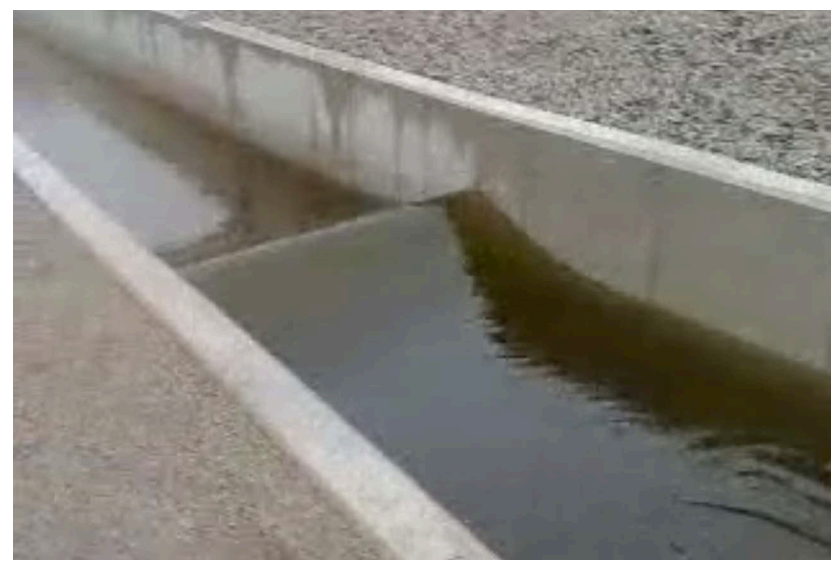

Fig. 1. Bore-soliton-splash experiment: a smooth soliton is generated just after the sluice gate has been opened. Run case 6 (Zweers, 2010).

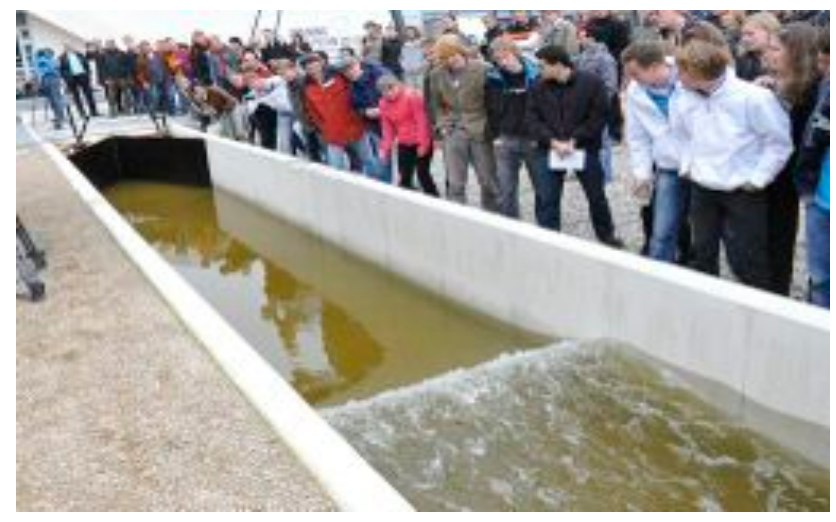

Fig. 2. Bore-soliton-splash experiment: after the soliton breaks it propagates as a bore through the channel. Run case 8. Photo: University of Twente (Bokhove et al., 2011).

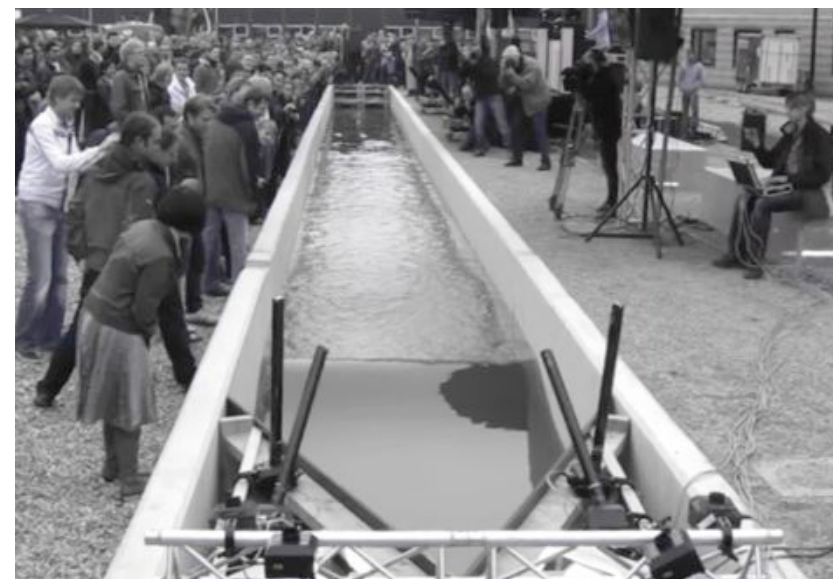

Fig. 3. Bore-soliton-splash experiment: the broken wave has dissipated enough energy near the end of the channel such that it is smoothed back to a soliton of lower amplitude. Run case 8 (Bokhove et al., 2011). 


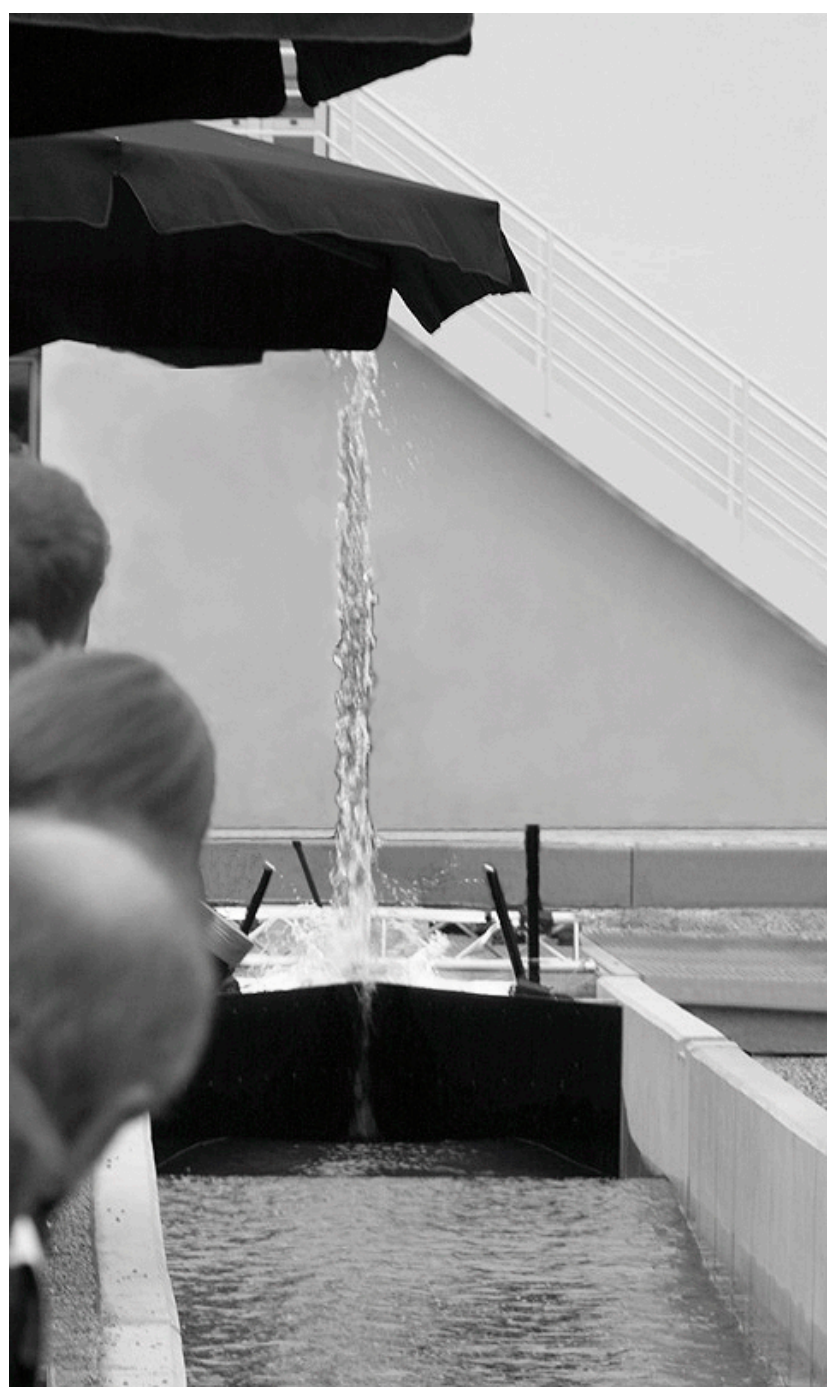

Fig. 4. Bore-soliton-splash experiment: the final splash in the constriction of the channel. Run case 8 (Zweers, 2010).

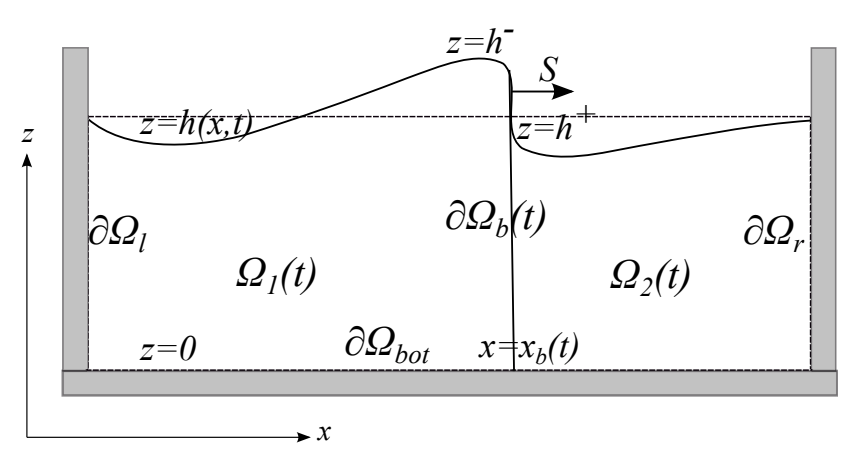

Fig. 5. Domain sketch for a breaking wave. Vertical cut with axes $(x, z)$. corresponding depth-averaged horizontal velocity $u=\phi_{x} \equiv$ $\partial_{x} \phi$. The analog of the extended Luke's variational principle (2)-(5) for the depth-averaged shallow water system is

$$
\begin{aligned}
0 & =\delta \int_{0}^{T} \mathcal{L}[\phi, h, t] \mathrm{d} t \\
& =\delta \int_{0}^{T}\left(\int_{0}^{x_{\mathrm{b}}^{-}}\left(-h \phi_{t}-\frac{1}{2} h\left(\phi_{x}\right)^{2}-\frac{1}{2} g h^{2}+g h H_{0}\right) \mathrm{d} x\right. \\
& \left.+\int_{x_{\mathrm{b}}^{+}}^{L}\left(-h \phi_{t}-\frac{1}{2} h\left(\phi_{x}\right)^{2}-\frac{1}{2} g h^{2}+g h H_{0}\right) \mathrm{d} x\right) \mathrm{d} t,
\end{aligned}
$$

in which we used the more compact notation $\phi_{t} \equiv \partial_{t} \phi$, etc., and $x_{\mathrm{b}}^{-}=\lim _{\epsilon \rightarrow 0^{-}}\left(x_{\mathrm{b}}+\epsilon\right)$ and $x_{\mathrm{b}}^{+}=\lim _{\epsilon \rightarrow 0^{+}}\left(x_{\mathrm{b}}+\epsilon\right)$.

Taking variations of (63) we get

$$
\begin{aligned}
0 & =\delta \int_{0}^{T} \mathcal{L}[\phi, h, t] \mathrm{d} t \\
& =\int_{0}^{T}\left(\int_{0}^{x_{\mathrm{b}}^{-}}+\int_{x_{\mathrm{b}}^{+}}^{L}\left(\left(-\phi_{t}-\frac{1}{2}\left(\phi_{x}\right)^{2}-g h+g H_{0}\right) \delta h\right.\right. \\
& +\left(-h \partial_{t} \phi-\frac{1}{2} h\left(\phi_{x}\right)^{2}-\frac{1}{2} g h^{2}+g h H_{0}\right)^{-} \delta x_{b} \\
& -\left(-h \phi_{t}-h \phi_{x} \delta \phi_{x}\right) \mathrm{d} x \\
& \left.\left(-\frac{1}{2} h\left(\phi_{x}\right)^{2}-\frac{1}{2} g h^{2}+g h H_{0}\right)^{+} \delta x_{b}\right) \mathrm{d} t .
\end{aligned}
$$

When we work out some terms in detail, we obtain

$$
\begin{aligned}
& \int_{0}^{T}\left(\int_{0}^{x_{\mathrm{b}}^{-}}+\int_{x_{\mathrm{b}}^{+}}^{L} h \delta \phi_{t} \mathrm{~d} x \mathrm{~d} t=\right. \\
& \int_{0}^{T}\left(\int_{0}^{x_{\mathrm{b}}^{-}}+\int_{x_{\mathrm{b}}^{+}}^{L}\left((h \delta \phi)_{t}-h_{t} \delta \phi\right) \mathrm{d} x\right) \mathrm{d} t \\
& =\int_{0}^{T}\left(\int_{0}^{x_{\mathrm{b}}^{-}} \int_{x_{\mathrm{b}}^{+}}^{L}+h_{t} \delta \phi \mathrm{d} x+\frac{\mathrm{d}}{\mathrm{d} t}\left(\left(\int_{0}^{x_{b}^{-}} \int_{x_{\mathrm{b}}^{+}}^{L}\right) h \delta \phi \mathrm{d} x\right)\right. \\
& \left.\quad-\dot{x}_{\mathrm{b}} h^{-}(\delta \phi)^{-}+\dot{x}_{\mathrm{b}} h^{+}(\delta \phi)^{+}\right) \mathrm{d} t,
\end{aligned}
$$


and

$$
\begin{aligned}
& \int_{0}^{T}\left(\int_{0}^{x_{\mathrm{b}}^{-}}+\int_{x_{\mathrm{b}}^{+}}^{L} h \phi_{x} \delta \phi_{x} \mathrm{~d} x\right) \mathrm{d} t \\
& =\int_{0}^{T}\left(\left(\int_{0}^{x_{\mathrm{b}}^{-}}+\int_{x_{\mathrm{b}}^{+}}^{L}\right)\left(\left(h \phi_{x} \delta \phi\right)_{x}-\left(h \phi_{x}\right)_{x} \delta \phi\right) \mathrm{d} x\right) \mathrm{d} t \\
& =\int_{0}^{T}\left(-\left(\int_{0}^{x_{\mathrm{b}}^{-}}+\int_{x_{\mathrm{b}}^{+}}^{L}\right)\left(h \phi_{x}\right)_{x} \delta \phi \mathrm{d} x\right. \\
& \left.+\left(h \phi_{x} \delta \phi\right)^{-}-\left(h \phi_{x} \delta \phi\right)^{+}\right) \mathrm{d} t .
\end{aligned}
$$

Using the endpoint conditions $(\delta \phi)_{t=0}=(\delta \phi)_{t=T}$, $(\delta h)_{t=0}=(\delta h)_{t=T}$, the resulting variations become

$$
\begin{aligned}
0 & =\delta \int_{0}^{T} \mathcal{L}[\phi, h, t] \mathrm{d} t \\
& =\int_{0}^{T}\left(\int_{0}^{x_{b}^{-}}+\int_{x_{b}^{+}}^{L}\right)\left(\left(-\phi_{t}-\frac{1}{2}\left(\phi_{x}\right)^{2}-g h+g H_{0}\right) \delta h\right. \\
& \left.+\left(h_{t}+\left(h \phi_{x}\right)_{x}\right) \delta \phi\right) \mathrm{d} x \\
& +\left[h \phi_{t}+\frac{1}{2} h\left(\phi_{x}\right)^{2}+\frac{1}{2} g h^{2}-g h H_{0}\right] \delta x_{b} \\
& +S h^{-}(\delta \phi)^{-}-S h^{+}(\delta \phi)^{+}-\left(h \phi_{x} \delta \phi\right)^{-} \\
& \left.+\left(h \phi_{x} \delta \phi\right)^{+}\right) \mathrm{d} t,
\end{aligned}
$$

where we defined the jump $[f] \equiv f^{+}-f^{-}$for an arbitrary quantity $f$. Under the assumption that the velocity field can at most contain discontinuities, it follows that the velocity potential at the interface $\phi_{\mathrm{b}}=\phi\left(x_{\mathrm{b}}\right)=\phi\left(x_{\mathrm{b}}^{-}\right)=\phi\left(x_{\mathrm{b}}^{+}\right)$must be continuous. For variations over the interface variables we use the relation $\delta\left(\phi_{\mathrm{b}}\right)=(\delta \phi)_{\mathrm{b}}+\left(\phi_{x}\right)_{\mathrm{b}} \delta x_{\mathrm{b}}$, and then obtain

$$
\begin{aligned}
0 & =\delta \int_{0}^{T} \mathcal{L}[\phi, h, t] \mathrm{d} t \\
& =\int_{0}^{T}\left(\int_{0}^{x_{\mathrm{b}}^{-}}+\int_{x_{\mathrm{b}}^{+}}^{L}\right)\left(\left(-\phi_{t}-\frac{1}{2}\left(\phi_{x}\right)^{2}-g h+g H_{0}\right) \delta h\right. \\
& \left.+\left(h_{t}+\left(h \phi_{x}\right)_{x}\right) \delta \phi\right) \mathrm{d} x \\
& +\left[h \phi_{t}+\frac{1}{2} h\left(\phi_{x}\right)^{2}+\frac{1}{2} g h^{2}-g h H_{0}\right] \delta x_{\mathrm{b}} \\
& \left.-\left[S h-h \phi_{x}\right] \delta \phi_{\mathrm{b}}+\left[S h \phi_{x}-h \phi_{x}^{2}\right] \delta x_{\mathrm{b}}\right) \mathrm{d} t .
\end{aligned}
$$

The final step is to use the equation for $\phi_{t}$ following from the arbitrary variation $\delta h$ to combine the terms with $\delta x_{\mathrm{b}}$.

Subsequently, variations with respect to $\phi, h, x_{\mathrm{b}}, \phi_{\mathrm{b}}$ produce the following system of equations

$$
\begin{aligned}
\delta \phi: & \partial_{t} h+\left(h \phi_{x}\right)_{x}=0, \text { at }\left[0, x_{\mathrm{b}}^{-}\right) \cup\left(x_{\mathrm{b}}^{+}, L\right], \\
\delta h: & \partial_{t} \phi+\frac{1}{2}\left(\phi_{x}\right)^{2}+g\left(h-H_{0}\right)=0, \\
& \text { at }\left[0, x_{\mathrm{b}}^{-}\right) \cup\left(x_{\mathrm{b}}^{+}, L\right],
\end{aligned}
$$

$\delta \phi_{\mathrm{b}}: \quad\left[h\left(S-\phi_{x}\right)\right]=0, \quad$ at $x=x_{\mathrm{b}}$,

$\delta x_{\mathrm{b}}:\left[h \phi_{x}^{2}-h S \phi_{x}+\frac{1}{2} g h^{2}\right]=0, \quad$ at $x=x_{\mathrm{b}}$.

Equations (69a)-(69b) are the well-known shallow water equations. Using $u=\phi_{x}$ they can be represented as

$\partial_{t} h+(h u)_{x}=0 \quad$ and $\quad \partial_{t} u+u u_{x}+g h_{x}=0$,

in $\left[0, x_{\mathrm{b}}^{-}\right) \cup\left(x_{\mathrm{b}}^{+}, L\right]$, and the jump conditions $(69 \mathrm{c})$ and $(69 \mathrm{~d})$ are reformulated as

$\delta \phi_{\mathrm{b}}: \quad[h(S-u)]=0$,

$\delta x_{\mathrm{b}}: \quad\left[h(S-u)^{2}+\frac{1}{2} g h^{2}\right]=0$.

These are the well-known Rankine-Hugoniot conditions for a moving bore in the shallow water equations.

An important property of hydraulic jumps in shallow water is the loss of energy similar to the rise of entropy for shocks in compressible fluid dynamics. It corresponds to the observation that breaking waves spray into many droplets losing mechanical energy in the turbulent processes. The energy of the system is given by the Hamiltonian. Taking the time derivative of the Hamiltonian and using (70), we obtain

$$
\begin{aligned}
\frac{\mathrm{d} \mathcal{H}}{\mathrm{d} t}= & \left.\frac{\mathrm{d}}{\mathrm{d} t} \int_{0}^{x_{\mathrm{b}}^{-}}+\int_{x_{\mathrm{b}}^{+}}^{L}\right)\left(\frac{1}{2} h u^{2}+\frac{1}{2} g h^{2}\right) \mathrm{d} x \\
= & \left(\int_{0}^{x_{\mathrm{b}}^{-}}+\int_{x_{\mathrm{b}}^{+}}^{L}\right)\left(\frac{1}{2} h u^{2}+\frac{1}{2} g h^{2}\right)_{t} \mathrm{~d} x \\
= & \left(\int_{0}^{x_{\mathrm{b}}}\left[\frac{1}{2} h u^{2}+\frac{1}{2} g h^{2}\right]\right. \\
& -S\left[\frac{1}{x_{\mathrm{b}}^{+}}\right) \\
& -S\left[u^{3}-g h^{2} u\right)_{x} \mathrm{~d} x \\
= & {\left[\frac{1}{2} h u^{3}+g h^{2} u\right]-S\left[\frac{1}{2} h h^{2}\right] }
\end{aligned}
$$


which equals expression (13.86) in Whitham (1974). Using the jump conditions (71), the expression (72) takes the known form

$\frac{\mathrm{d} \mathcal{H}}{\mathrm{d} t}=\frac{g\left(h^{+}-h^{-}\right)^{3} h^{-}\left(S-u^{-}\right)}{4 h^{-} h^{+}}$,

which means that if $h^{+}>h^{-}$and $S-u^{+}<0$ or $h^{+}<h^{-}$ and $S-u^{+}>0$ energy is lost in (73). These cases can also be clarified by taking the velocity $u^{-}=0$, keeping the left domain at rest, when the bore comes. When $h^{+}>h^{-}$, the bore then must come from the right, and therefore $S<0$, whence the condition is satisfied. Vice versa when $u^{+}=0$, the right domain is at rest. When $h^{+}<h^{-}$, then the bore must come from the left and $S>0$.

\subsection{2-D jump conditions for new water wave model}

The jump conditions for the new water wave model in two horizontal dimensions (2DH) can be obtained in a similar way. The three dimensional domain $\Omega$ is split into two parts: $\Omega_{1}$ lying on one side of the bore and $\Omega_{2}$ is lying on the other side, see Fig. 6. Viewed from above, the maximum horizontal extents of these two domains at the free surface are denoted by $\Omega_{1 \mathrm{H}}$ and $\Omega_{2 \mathrm{H}}$, respectively. Between the domains there is a vertical and curved moving interface $\partial \Omega_{\mathrm{b}}$, corresponding to the unknown bore position $\left(x_{\mathrm{b}}, y_{\mathrm{b}}\right)(t)$ in the horizontal plane. The domain $\Omega$ is taken to have solid wall boundaries and a flat bottom. The free surface $z=h$ is denoted as $\partial \Omega_{\mathrm{s}}$. Assume that $h^{-}>h^{+}$with $h^{-}$and $h^{+}$along the interface $\partial \Omega_{\mathrm{b}}$ in $\Omega_{1}$ and $\Omega_{2}$, respectively. Hence, the bore moves towards $\Omega_{2}$ with speed $S=\left(\dot{x}_{\mathrm{b}}, \dot{y}_{\mathrm{b}}\right)^{T} \cdot \boldsymbol{n}$. We use $\boldsymbol{n}$ for the outward normal of the domain $\Omega_{1}$ at the point $\mathbf{x}_{\mathrm{b}}=\left(x_{\mathrm{b}}, y_{\mathrm{b}}\right)^{T}$ along the moving boundary $\partial \Omega_{\mathrm{b}}$. At the same point $\mathbf{x}_{\mathrm{b}}$ the outward normal $\boldsymbol{n}_{2}$ in $\Omega_{2}$ has the opposite sign: $\boldsymbol{n}_{2}=-\boldsymbol{n}$. In the expressions that follow, we generally omit the third zero component of this normal at the vertical boundary $\partial \Omega_{\mathrm{b}}$.

The velocity field in the new water wave model has the form

$\boldsymbol{U}(x, y, z, t)=\nabla \phi+\pi_{i} \nabla \boldsymbol{l}_{i}=\nabla \phi+\mathbf{v}=\boldsymbol{u}^{*}+\nabla \varphi$,

with $i=1,2$. The depth-averaged horizontal velocity is defined as

$\overline{\boldsymbol{u}}(x, y, t)=\frac{1}{h} \int_{0}^{h} \boldsymbol{U}_{\mathrm{H}} \mathrm{d} z$.

As before in Eq. (2), the variational principle for the new

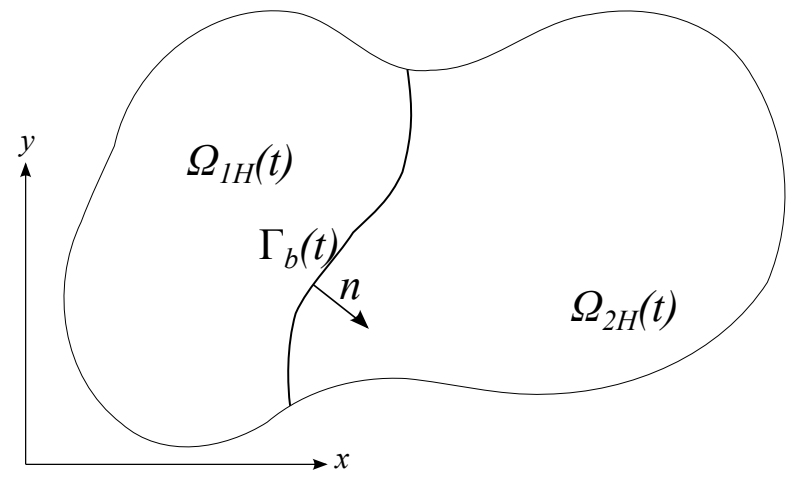

Fig. 6. Domain sketch for a breaking wave. Horizontal cut with axes $(x, y)$. The bore boundary $\partial \Omega_{\mathrm{b}}$ is a vertical sheet with bore line $\Gamma_{\mathrm{b}}$.

water wave model has the form

$$
\begin{aligned}
& 0=\delta \int_{0}^{T} \mathcal{L}\left[\boldsymbol{l}, \boldsymbol{\pi}, \boldsymbol{\phi}, \boldsymbol{\phi}_{\mathrm{S}}, h\right] \mathrm{d} t \\
& =\delta \int_{0}^{T}\left(\int_{\Omega_{1 \mathrm{H}}} \int_{0}^{h}\left(\partial_{t} \phi+\boldsymbol{\pi} \cdot \partial_{t} \boldsymbol{l}+\frac{1}{2}|\boldsymbol{U}|^{2}+g\left(z-H_{0}\right)\right) \mathrm{d} z \mathrm{~d} x \mathrm{~d} y\right. \\
& \left.+\int_{\Omega_{2 \mathrm{H}}} \int_{0}^{h}\left(\partial_{t} \phi+\boldsymbol{\pi} \cdot \partial_{t} \boldsymbol{l}+\frac{1}{2}|\boldsymbol{U}|^{2}+g\left(z-H_{0}\right)\right) \mathrm{d} z \mathrm{~d} x \mathrm{~d} y\right) \mathrm{d} t .
\end{aligned}
$$

First, we have to identify the independent variables, with respect to which we take the variations. Clearly, these include $\phi$ in the interior, and $h, \phi_{\mathrm{s}}, \boldsymbol{l}$ and $\pi$ at the free surface. Again, we impose the following continuity assumptions on the velocity potential, $\phi_{\mathrm{b}}=\phi\left(x_{\mathrm{b}}, y_{\mathrm{b}}, z, t\right)=\phi\left(x_{\mathrm{b}}^{-}, y_{\mathrm{b}}^{-}, z, t\right)=$ $\phi\left(x_{\mathrm{b}}^{+}, y_{\mathrm{b}}^{+}, z, t\right)$, and the particle labels, $\boldsymbol{l}_{\mathrm{b}}=\boldsymbol{l}\left(x_{\mathrm{b}}, y_{\mathrm{b}}, t\right)=$ $\boldsymbol{l}\left(x_{\mathrm{b}}^{-}, y_{\mathrm{b}}^{-}, t\right)=\boldsymbol{l}\left(x_{\mathrm{b}}^{+}, y_{\mathrm{b}}^{+}, t\right)$, at the bore boundary $\partial \Omega_{\mathrm{b}}$. It turns out later that $\boldsymbol{l}_{\mathrm{b}}$ and $\phi_{\mathrm{b}}$ emerge as independent variations as well. The interior boundary is evolving in time, implying that $x_{\mathrm{b}}(q, t)$ and $y_{\mathrm{b}}(q, t)$ are part of the dynamics for some parameterization involving $q$ along $\partial \Omega_{\mathrm{b}}$. It turns out to be more convenient to work in a coordinate system along $\partial \Omega_{\mathrm{b}}$ that is aligned with the normal vector $\boldsymbol{n}$ and tangential vector $\boldsymbol{\tau}$ tangential to it, such that $\boldsymbol{n} \cdot \boldsymbol{\tau}=0$. By definition this normal coincides with the direction of the jump with speed $S$. Instead of $\delta \mathbf{x}_{\mathrm{b}}$ we will use $\left(\delta n_{\mathrm{b}}, \delta \tau_{\mathrm{b}}\right)^{T}$, concerning the variations of a bore position in the $\boldsymbol{n}$ and $\boldsymbol{\tau}$ directions. The projection of the vector $\delta \mathbf{x}_{\mathrm{b}}$ on the normal $\boldsymbol{n}$ is $\delta n_{\mathrm{b}}=\left(\delta x_{\mathrm{b}}, \delta y_{\mathrm{b}}\right)^{T} \cdot \boldsymbol{n}$. Similarly, $\delta \tau_{\mathrm{b}}=\left(\delta x_{\mathrm{b}}, \delta y_{\mathrm{b}}\right)^{T} \cdot \boldsymbol{\tau}$, with $\boldsymbol{n}=\left(n_{1}, n_{2}\right)^{T}$ and $\boldsymbol{\tau}=\left(\tau_{1}, \tau_{2}\right)^{T}$ the unit vectors of the new coordinate system. Hence, we find

$\delta x_{\mathrm{b}}=n_{1} \delta n_{\mathrm{b}}+\tau_{1} \delta \tau_{\mathrm{b}}$,
$\delta y_{\mathrm{b}}=n_{2} \delta n_{\mathrm{b}}+\tau_{2} \delta \tau_{\mathrm{b}}$. 
Using (77), we relate variations of $\phi$ at the boundary $\partial \Omega_{\mathrm{b}}$, as follows:

$$
\begin{aligned}
\delta\left(\phi_{\mathrm{b}}\right) & =(\delta \phi)_{\mathrm{b}}+\left(\phi_{x}\right)_{\mathrm{b}} \delta x_{\mathrm{b}}+\left(\phi_{y}\right)_{\mathrm{b}} \delta y_{\mathrm{b}} \\
& =(\delta \phi)_{\mathrm{b}}+\left(n_{1} \phi_{x}+n_{2} \phi_{y}\right)_{\mathrm{b}} \delta n_{\mathrm{b}}+\left(\tau_{1} \phi_{x}+\tau_{2} \phi_{y}\right)_{\mathrm{b}} \delta \tau_{\mathrm{b}} \\
& =(\delta \phi)_{\mathrm{b}}+(\nabla \phi \cdot \boldsymbol{n})_{\mathrm{b}} \delta n_{\mathrm{b}}+(\nabla \phi \cdot \boldsymbol{\tau})_{\mathrm{b}} \delta \tau_{\mathrm{b}} .
\end{aligned}
$$

Similar formulas can be obtained, as follows:

$$
\begin{aligned}
& \delta\left(h^{+}\right)=(\delta h)^{+}+(\nabla h)^{+} \cdot \boldsymbol{n} \delta n_{\mathrm{b}}+(\nabla h)^{+} \cdot \boldsymbol{\tau} \delta \tau_{\mathrm{b}}, \\
& \frac{\mathrm{d} h^{+}}{\mathrm{d} t}=\left(h_{t}\right)^{+}+(\nabla h)^{+} \cdot \boldsymbol{n} S, \\
& \frac{\mathrm{d} \phi_{\mathrm{b}}}{\mathrm{d} t}=\left(\phi_{t}\right)_{\mathrm{b}}+(\nabla \phi)_{\mathrm{b}} \cdot \boldsymbol{n} S .
\end{aligned}
$$

The subdomains $\Omega_{i}$ with $i=1,2$ are time-dependent, since the interface between these subdomains moves in time. We therefore have to use a variational analogue of Reynolds' transport theorem (see e.g. Daniljuk, 1976; Flanders, 1973, and Appendix B), as follows:

$\delta \int_{\Omega_{i}} F \mathrm{~d} x \mathrm{~d} y \mathrm{~d} z=\int_{\partial \Omega_{i}} F \delta \mathbf{x}_{\Gamma} \cdot \boldsymbol{n}_{\Gamma} \mathrm{d} \Gamma_{i}+\int_{\Omega_{i}} \delta F \mathrm{~d} x \mathrm{~d} y \mathrm{~d} z$,

with the time-dependent part of the boundary $\Gamma_{i}, \boldsymbol{n}_{\Gamma}$ the three dimensional normal to the boundary, and $\delta \mathbf{x}_{\Gamma}=$ $\left(\delta x_{\Gamma}, \delta y_{\Gamma}, \delta z_{\Gamma}\right)^{T}$ the variations of the coordinates of that boundary. Given (76), the expression for $F$ is complicated and depends on the independent variables in the variational principle. It may in principle also contain given functions of space, such as the bottom topography $b=b(x, y)$ (here set to zero for simplicity). There are two time-dependent parts of the subdomains $\Omega_{i}$ in (76): the 2-D free surface $\Gamma_{\mathrm{s}}$ and the bore boundary $\partial \Omega_{\mathrm{b}}$, which extends from $z \in[0, h]$ along the horizontal 1-D bore line $\Gamma_{\mathrm{b}}$. The outward normal at the free surface is $\mathbf{n}_{\mathrm{s}}=\left(-h_{x},-h_{y}, 1\right)^{T} / \sqrt{1+\left(h_{x}\right)^{2}+\left(h_{y}\right)^{2}}$. The chosen parameterization is $z-h(x, y, t)=0$ for a singlevalued free surface, in which $x$ and $y$ are the coordinates. In addition, $\mathrm{d} \Gamma_{\mathrm{S}}=\sqrt{1+\left(h_{x}\right)^{2}+\left(h_{y}\right)^{2}} \mathrm{~d} x \mathrm{~d} y$. Hence,

$$
\begin{aligned}
\delta \mathbf{x}_{\mathrm{s}} \cdot \boldsymbol{n}_{\mathrm{s}} \mathrm{d} \Gamma_{\mathrm{s}} & =\left(\delta x_{\mathrm{s}} \delta y_{\mathrm{s}}, \delta z_{\mathrm{s}}\right)^{T} \cdot\left(-h_{x},-h_{y}, 1\right) \mathrm{d} x \mathrm{~d} y \\
& =\delta h \mathrm{~d} x \mathrm{~d} y,
\end{aligned}
$$

since $\delta x_{\mathrm{s}}=\delta x=0, \delta y_{\mathrm{s}}=\delta y=0$ and $\delta z_{\mathrm{s}}=\delta h$. The bore boundary is vertical and a line when viewed from above with parameterization $x_{\mathrm{b}}(q, t)$ and $y_{\mathrm{b}}(q, t)$ with parameter $q$ along this line. The tangential vector $\boldsymbol{\tau}=\left(x_{q}, y_{q}, 0\right)^{T}$ with $x_{q} \equiv \partial_{q} x_{\mathrm{b}}$ and $y_{q} \equiv \partial_{q} y_{\mathrm{b}}$. Hence the three-dimensional normal is $\boldsymbol{n}=\left(-y_{q}, x_{q}, 0\right)^{T}$ in the direction of bore propagation. Consequently, Reynolds' theorem for variations used here becomes

$$
\begin{aligned}
& \delta \int_{\Omega_{i}} F \mathrm{~d} x \mathrm{~d} y \mathrm{~d} z=\int_{\Gamma_{\mathrm{s}}} F \delta h \mathrm{~d} x \mathrm{~d} y \\
& \quad+\int_{0}^{h} \int_{\Gamma_{\mathrm{b}}} F \delta \mathbf{x}_{\mathrm{b}} \cdot \boldsymbol{n}_{\mathrm{b}} \mathrm{d} \Gamma_{\mathrm{b}} \mathrm{d} z+\int_{\Omega_{i}} \delta F \mathrm{~d} x \mathrm{~d} y \mathrm{~d} z
\end{aligned}
$$

with $d \Gamma_{\mathrm{b}}$ an infinitesimal line element along the bore line $\Gamma_{\mathrm{b}}$.

Reynolds' transport theorem for time derivatives has a similar form as (82) provided we change the variational derivatives by time derivatives, giving

$$
\begin{aligned}
& \frac{\mathrm{d}}{\mathrm{d} t} \int_{\Omega_{i}} F \mathrm{~d} x \mathrm{~d} y \mathrm{~d} z=\int_{\Gamma_{\mathrm{s}}} F \partial_{t} h \mathrm{~d} x \mathrm{~d} y \\
& \quad+\int_{0}^{h} \int_{\Gamma_{\mathrm{b}}} F S \mathrm{~d} \Gamma_{\mathrm{b}} \mathrm{d} z+\int_{\Omega_{i}} \partial_{t} F \mathrm{~d} x \mathrm{~d} y \mathrm{~d} z .
\end{aligned}
$$

Application of (82) to the variations in (76) yields

$$
\begin{aligned}
0= & \int_{0}^{T} \int_{\Omega_{1 \mathrm{H}}, \Omega_{2 \mathrm{H}}}\left(\partial_{t} \phi+\boldsymbol{\pi} \cdot \partial_{t} \boldsymbol{l}+\frac{1}{2}|\boldsymbol{U}|^{2}+g\left(h-H_{0}\right)\right)_{\mathrm{s}} \delta h \mathrm{~d} x \mathrm{~d} y \\
-\int_{\Gamma_{\mathrm{b}}} & {\left[\int_{0}^{h}\left(\partial_{t} \phi+\pi \cdot \partial_{t} \boldsymbol{l}+\frac{1}{2}|\boldsymbol{U}|^{2}\right) \mathrm{d} z+\frac{g h^{2}}{2}-g h H_{0}\right] \delta n_{\mathrm{b}} \mathrm{d} \Gamma_{\mathrm{b}} } \\
+ & \int_{\Omega_{1 \mathrm{H}}, \Omega_{2 \mathrm{H}}} \int_{0}^{h} \delta \phi_{t}+\boldsymbol{U} \cdot \delta(\nabla \phi)+\boldsymbol{\pi}_{i} \boldsymbol{U} \cdot \delta\left(\nabla \boldsymbol{l}_{i}\right) \\
& +\boldsymbol{U} \cdot \nabla \boldsymbol{l}_{i} \delta \boldsymbol{\pi}_{i} \mathrm{~d} z+h \boldsymbol{l}_{t} \cdot \delta \boldsymbol{\pi}+h \boldsymbol{\pi} \cdot \delta \boldsymbol{l}_{t} \mathrm{~d} x \mathrm{~d} y \mathrm{~d} t
\end{aligned}
$$

with the jump notation $[F]=F^{+}-F^{-}$for some arbitrary quantity $F$ across the bore.

We illustrate the derivation by working out one of the variations in detail. Using Reynolds' transport theorems (82) and (83), the variation of the integrals involving $\phi_{t}$ in (76) (see also Eq. 84) becomes

$$
\begin{aligned}
& \delta \int_{0}^{T} \int_{\Omega_{1 \mathrm{H}}, \Omega_{2 \mathrm{H}}} \int_{0}^{h} \phi_{t} \mathrm{~d} z \mathrm{~d} x \mathrm{~d} y \mathrm{~d} t \\
& =\int_{0}^{T} \int_{\Gamma_{\mathrm{S}}}\left(\phi_{t}\right)_{\mathrm{s}} \delta h \mathrm{~d} x \mathrm{~d} y+\iint_{\Gamma_{\mathrm{b}}}^{h}\left[-\phi_{t}\right] \delta n_{\mathrm{b}} \mathrm{d} z \mathrm{~d} \Gamma_{\mathrm{b}} \\
& +\frac{\mathrm{d}}{\mathrm{d} t} \int_{\Omega_{1 \mathrm{H}}, \Omega_{2 \mathrm{H}}} \int_{0}^{h} \delta \phi \mathrm{d} z \mathrm{~d} x \mathrm{~d} y-\int_{\Gamma_{\mathrm{S}}} h_{t}(\delta \phi)_{\mathrm{s}} \mathrm{d} x \mathrm{~d} y \\
& -\int_{\Gamma_{\mathrm{b}}} S\left[\int_{0}^{h}(\delta \phi)_{\mathrm{b}} \mathrm{d} z\right] \mathrm{d} \Gamma_{\mathrm{b}} \mathrm{d} t .
\end{aligned}
$$


In the last step, we use the end-point conditions $(\delta \phi)_{t=0}=$ $(\delta \phi)_{t=T}=0$, expression (78) for $(\delta \phi)_{\mathrm{b}}$ and a similar expression for $(\delta \phi)_{\mathrm{s}}=\delta\left(\phi_{\mathrm{s}}\right)-\left(\phi_{z}\right)_{\mathrm{s}} \delta h$, in order to determine the variations with respect to the independent variables. The variations in the last two terms in (85) thus become

$$
\begin{aligned}
& =\int_{0}^{T}-\int_{\Gamma_{\mathrm{s}}} h_{t}\left(\delta\left(\phi_{\mathrm{s}}\right)-\left(\phi_{z}\right)_{\mathrm{s}} \delta h\right) \mathrm{d} x \mathrm{~d} y \\
& -\int_{\Gamma_{\mathrm{b}}}\left[S \int_{0}^{h}\left(\delta\left(\phi_{b}\right)-\nabla \phi \cdot \boldsymbol{n} \delta n_{b}-\nabla \phi \cdot \boldsymbol{\tau} \delta \tau_{b}\right) \mathrm{d} z\right] \mathrm{d} \Gamma_{\mathrm{b}} \mathrm{d} t .
\end{aligned}
$$

Analysing the variations of (84) in a similar way, while integrating by parts, using the endpoint conditions $(\delta \phi)_{t=0}=$ $(\delta \phi)_{t=T},(\delta \boldsymbol{l})_{t=0}=(\delta \boldsymbol{l})_{t=T}$, and the definition of the velocity (74), we first obtain the following system of equations

$$
\begin{aligned}
& \delta \phi: \nabla^{2} \phi+\nabla \cdot \mathbf{v}=0 \quad \text { in } \quad \Omega \backslash \partial \Omega_{\mathrm{b}}, \\
& \delta h: \partial_{t} \phi_{\mathrm{s}}+\frac{1}{2}\left|\nabla_{\mathrm{H}} \phi_{\mathrm{s}}+\mathbf{v}\right|^{2}+g\left(h-H_{0}\right)-\mathbf{v} \cdot \overline{\boldsymbol{u}} \\
&-\frac{1}{2}\left(\partial_{z} \phi\right)_{\mathrm{s}}^{2}\left(1+\left|\nabla_{\mathrm{H}} h\right|^{2}\right)=0 \quad \text { in } \quad \Gamma_{\mathrm{s}} \backslash \Gamma_{\mathrm{b}}, \\
& \delta \phi_{\mathrm{s}}: \partial_{t} h-\left(\partial_{z} \phi\right)_{\mathrm{s}}\left(1+\left|\nabla_{\mathrm{H}} h\right|^{2}\right) \\
&+\left(\nabla_{\mathrm{H}} \phi_{\mathrm{s}}+\mathbf{v}\right) \cdot \nabla_{\mathrm{H}} h=0 \quad \text { in } \quad \Gamma_{\mathrm{s}} \backslash \Gamma_{\mathrm{b}}, \\
& \delta(h \boldsymbol{\pi}): \quad \partial_{t} \boldsymbol{l}+\overline{\boldsymbol{u}} \cdot \nabla \boldsymbol{l}=0 \quad \text { in } \quad \Gamma_{\mathrm{s}} \backslash \Gamma_{\mathrm{b}}, \\
& \delta \boldsymbol{l}: \quad \partial_{t}(h \boldsymbol{\pi})+\nabla \cdot(h \overline{\boldsymbol{u}} \boldsymbol{\pi})=0 \quad \text { in } \quad \Gamma_{\mathrm{s}} \backslash \Gamma_{\mathrm{b}},
\end{aligned}
$$

similar to (6) and (7). It can also be reformulated to (27) and (28).

Second, the variations with respect to the interior boundary variables at $\partial \Omega_{\mathrm{b}}$ arise with the help of relations (78) and (79), and equations (87):

$$
\begin{aligned}
\delta \phi_{\mathrm{b}}: & {[h(\overline{\boldsymbol{u}} \cdot \boldsymbol{n}-S)]=0, } \\
\delta n_{\mathrm{b}}: & {\left[\int_{0}^{h}(\boldsymbol{U} \cdot \boldsymbol{n})(\boldsymbol{U} \cdot \boldsymbol{n}-S) \mathrm{d} z-\frac{1}{2} g h^{2}\right.} \\
& \left.\int_{0}^{h}\left(\phi_{t}+\frac{1}{2}|\boldsymbol{U}|^{2}\right) \mathrm{d} z+h \mathbf{v} \cdot \overline{\boldsymbol{u}}\right]=0, \\
\delta \tau_{\mathrm{b}}: \quad & {\left[\int_{0}^{h}(\boldsymbol{U} \cdot \boldsymbol{\tau})(\boldsymbol{U} \cdot \boldsymbol{n}-S) \mathrm{d} z\right]=0, } \\
\delta \boldsymbol{l}_{\mathrm{b}}: \quad & {[h \boldsymbol{\pi}(\overline{\boldsymbol{u}} \cdot \boldsymbol{n}-S)]=0 . }
\end{aligned}
$$

Together with (88a), condition (88d) expresses continuity of the Lagrange multipliers $\pi$. The jump conditions (88a) and (88b) coincide with the jump conditions resulting from the conservative form (33) of the new water wave model, and the jump condition (88c) shows that the tangential component of velocity contains no jump.

The expected loss of energy can be found via a similar procedure as for the shallow water equations. Taking the time derivative of the Hamiltonian, invoking Reynolds' theorem (83), and using (87) extensively, we find

$$
\begin{aligned}
\frac{\mathrm{d} \mathcal{H}}{\mathrm{d} t}= & \frac{\mathrm{d}}{\mathrm{d} t} \int_{\Omega_{1 \mathrm{H}}, \Omega_{2 \mathrm{H}}}\left(\int_{0}^{h} \frac{1}{2}|\boldsymbol{U}|^{2} \mathrm{~d} z+\frac{1}{2} g h^{2}\right) \mathrm{d} x \mathrm{~d} y \\
= & \int_{\Omega_{1 \mathrm{H}}, \Omega_{2 \mathrm{H}}}\left(\int_{0}^{h} \frac{1}{2}|\boldsymbol{U}|^{2} \mathrm{~d} z+\frac{1}{2} g h^{2}\right) \mathrm{d} x \mathrm{~d} y \\
& -\int_{\Gamma_{\mathrm{b}}} S\left[\int_{0}^{h} \frac{1}{2}|\boldsymbol{U}|^{2} \mathrm{~d} z+\frac{1}{2} g h^{2}\right] \mathrm{d} \Gamma_{\mathrm{b}} \\
= & -\int_{\Gamma_{\mathrm{b}}}\left[\int_{0}^{h} \boldsymbol{U} \cdot \boldsymbol{n} \phi_{t} \mathrm{~d} z+(h \overline{\boldsymbol{u}} \cdot \boldsymbol{n})(\mathbf{v} \cdot \overline{\boldsymbol{u}})\right] \mathrm{d} \Gamma_{\mathrm{b}} \\
& -\int_{\Gamma_{\mathrm{b}}}\left[\int_{0}^{h} \frac{1}{2}|\boldsymbol{U}|^{2} \mathrm{~d} z+\frac{1}{2} g h^{2}\right] \mathrm{d} \Gamma_{\mathrm{b}} .
\end{aligned}
$$

Additionally, we used a rewritten form of the continuity equation $\partial_{t} h+\nabla \cdot(h \overline{\boldsymbol{u}})=0$ and $\partial_{t} \mathbf{v}+\overline{\boldsymbol{u}} \cdot \nabla \mathbf{v}+\mathbf{v} \nabla \overline{\boldsymbol{u}}=0$. Using jump conditions (88), expression (89) finally takes the form

$$
\begin{aligned}
\frac{\mathrm{d} \mathcal{H}}{\mathrm{d} t} & =\int_{\Gamma_{\mathrm{b}}} Q \mathrm{~d} \Gamma_{\mathrm{b}} \\
& \equiv \int_{\Gamma_{\mathrm{b}}}\left[\int_{0}^{h}(S-\boldsymbol{U} \cdot \boldsymbol{n})\left(\phi_{t}+S(\boldsymbol{U} \cdot \boldsymbol{n})-\mathbf{v} \cdot \overline{\boldsymbol{u}}\right) \mathrm{d} z\right] \mathrm{d} \Gamma_{\mathrm{b}}
\end{aligned}
$$

with rate of energy loss $Q$ along the bore boundary.

\subsection{2-D jump conditions for shallow water equations}

The variational approach can be implemented for the 2-D shallow water equations. Nevertheless, the final result for the jump conditions coincides with the jump conditions derived from (88) under the simplification of the velocity potential. When we assume the velocity potential to be $\phi=\phi_{\mathrm{s}}$, the shallow water velocity field emerges as

$$
\begin{aligned}
\boldsymbol{U}(x, y, t) & =\overline{\boldsymbol{u}}=\boldsymbol{u}^{*}(x, y, t)=\nabla \phi_{\mathrm{S}}(x, y, t)+\mathbf{v}(x, y, t) \\
& =\nabla \phi_{\mathrm{S}}+\boldsymbol{\pi}_{i} \nabla \boldsymbol{l}_{i},
\end{aligned}
$$

with $i, j=1,2$. For the surface velocity potential $\phi_{z}=0$, which allows us to compute the integral in (88b) explicitly and to simplify equation (87b). We substitute (87b) into 
(88b), which leads to the Rankine-Hugoniot conditions for the shallow water equations at $\Gamma_{\mathrm{b}}$ :

$\delta \phi_{\mathrm{b}}: \quad[h(\overline{\boldsymbol{u}} \cdot \boldsymbol{n}-S)]=0$,

$\delta n_{\mathrm{b}}: \quad\left[h(\overline{\boldsymbol{u}} \cdot \boldsymbol{n})(\overline{\boldsymbol{u}} \cdot \boldsymbol{n}-S)+\frac{1}{2} g h^{2}\right]=0$,

$\delta \tau_{\mathrm{b}}: \quad[h(\overline{\boldsymbol{u}} \cdot \boldsymbol{\tau})(\overline{\boldsymbol{u}} \cdot \boldsymbol{n}-S)]=0$,

$\delta \boldsymbol{l}_{\mathrm{b}}: \quad[h \boldsymbol{\pi}(\overline{\boldsymbol{u}} \cdot \boldsymbol{n}-S)]=0$,

yielding again continuity of the Lagrange multipliers $\pi$.

Under the assumption that $\phi=\phi_{\mathrm{s}}$, while using the simplified version of (87b), the energy loss expression (90) reduces to the shallow water energy loss expression as follows:

$Q=\left[(\overline{\boldsymbol{u}} \cdot \boldsymbol{n})\left(\frac{1}{2} h|\overline{\boldsymbol{u}}|^{2}+g h^{2}\right)\right]-S\left[\frac{1}{2} h|\overline{\boldsymbol{u}}|^{2}+\frac{1}{2} g h^{2}\right]$.

Using jump conditions (92a) and (92b) expression (93) takes the well-known form (Peregrine, 1998)

$Q=\frac{g\left(h^{+}-h^{-}\right)^{3} h^{+}\left(S-\boldsymbol{n} \cdot \overline{\boldsymbol{u}}^{+}\right)}{4 h^{-} h^{+}}$,

which means that in the cases $h^{+}>h^{-}$and $\left(S-\boldsymbol{n} \cdot \overline{\boldsymbol{u}}^{+}\right)<0$ or $h^{+}<h^{-}$and $\left(S-\boldsymbol{n} \cdot \overline{\boldsymbol{u}}^{+}\right)>0$ the energy is lost. It is a natural 2-D generalization of relation (73).

\subsection{1-D jump conditions for new water wave model}

In one horizontal dimension the velocity field reduces to potential flow $\boldsymbol{U}=\nabla \phi$ with $\mathbf{v}=0$. We again split the domain $\Omega$ into two parts, $\Omega_{1}$ and $\Omega_{2}$ and in each of them the free surface profile is assumed single valued.

The jump conditions (88a) and (88b) in 1-D are reformulated as

$$
\begin{aligned}
{[h(S-\bar{u})] } & =0, \\
{\left[\int_{0}^{h}\left(\frac{1}{2} u^{2}-\frac{1}{2} w^{2}-\partial_{t} \phi\right) \mathrm{d} z-h \bar{u} S-\frac{1}{2} g h^{2}\right] } & =0,
\end{aligned}
$$

with vertical velocity component $w=\phi_{z}$.

It is worthwhile to mention that the jump conditions could also be obtained from (33). In 1-D the depth-averaged momentum equation in (33) takes the form

$$
\left(\int_{0}^{h} u \mathrm{~d} z\right)_{t}+\left(\int_{0}^{h}\left(\frac{1}{2} u^{2}-\frac{1}{2} w^{2}-\partial_{t} \phi\right) \mathrm{d} z-\frac{1}{2} g h^{2}\right)_{x}=0,
$$

which relates to the jump condition (95b).

The energy loss relation can be reduced from (90) to a form

$\frac{\mathrm{d} \mathcal{H}}{\mathrm{d} t}=\left[\int_{0}^{h}(S-u)\left(\phi_{t}+S u\right) \mathrm{d} z\right]$.
It is not clear what the sign of $\mathrm{d} \mathcal{H} / \mathrm{d} t$ is in the previous expression. The integral expressions can be simplified when we use the Ritz method to approximate the velocity potential, which is illustrated next.

\subsection{1-D jump conditions for variational Boussinesq and Green-Naghdi equations}

When we substitute the Green-Naghdi ansatz

$\phi=\phi_{\mathrm{s}}+\frac{1}{2}\left(h^{2}-z^{2}\right) \psi$

into jump condition (95b), we obtain

$\left[h \bar{u}^{2}-h S \bar{u}+\frac{1}{2} g h^{2}-\frac{h^{3}}{3}\left(\psi_{t}-\psi^{2}+\bar{u} \psi_{x}\right)+\beta \frac{h^{5}}{15} \psi_{x}^{2}\right]=0$

after using that $\partial_{t} u^{*}=\partial_{t x}\left(\phi_{\mathrm{s}}\right)=-B_{x}$ such that we can use $\partial_{t}\left(\phi_{\mathrm{s}}\right)=-B$ with $B$ given in (58b). To reconstruct the Green-Naghdi system we take $\beta=0$ and following (59a) take $\psi=\bar{u}_{x}$. Then jump conditions (95a) and (99) are simplified as

$$
\begin{aligned}
{[h(S-\bar{u})] } & =0, \\
{\left[h \bar{u}^{2}-h S \bar{u}+\frac{g h^{2}}{2}-\frac{h^{3}}{3}\left(\bar{u}_{x t}-\left(\bar{u}_{x}\right)^{2}+\bar{u} \bar{u}_{x x}\right)\right] } & =0 .
\end{aligned}
$$

Considering (97) with the velocity potential simplified as (98) and for $\beta=0$, we reformulate the entropy expression for energy loss as follows:

$\frac{\mathrm{d} \mathcal{H}}{\mathrm{d} t}=\left[h(\bar{u}-S)\left(\frac{(\bar{u}-S)^{2}}{2}+g h+\frac{h^{2} \bar{u}_{x}^{2}}{2}-\frac{h^{2}}{3}\left(\bar{u}_{x t}+\bar{u} \bar{u}_{x x}\right)\right)\right]$.

In order to check whether energy is lost in the jump we need to determine the sign of (101). The analysis of El et al. (2006) shows that due to the strong influence of dispersion in the Green-Naghdi model a discontinuity cannot be maintained. In that case, (101) reduces to $\mathrm{d} \mathcal{H} / \mathrm{d} t=0$ as there is no discontinuity. It is unclear at the moment whether the variational Boussinesq model for the case $\beta=1$ can maintain bores. This can in principle be checked in a numerical model with a shock fitting approach, such that numerical dissipation at the discontinuity is at least avoided. Such an investigation is left to future work possibly using results from Ali and Kalisch (2010).

\section{Conclusions}

A systematic derivation of a new Hamiltonian formulation for water waves was given starting from the variational principle (2). The new water wave model includes both waterwave dispersion and the vertical component of the vorticity by construction. It was pointed out by Bridges and Needham (2011) that the shallow water equations, or any Boussinesq 
system without proper circulation in the vertical plane, miss an instability they found in Benney's shallow water equations. It remains an open question to what extent this omission of horizontal vorticity components matters in the shallow water flows investigated here.

Subsequently, we showed how the new Hamiltonian formulation reduces to the classical shallow water and potential flow models. The new system could be simplified further to an extension of the variational Boussinesq models of Klopman et al. (2010), now including potential vorticity. For a parabolic potential flow profile, these Hamiltonian Boussinesq models were shown to contain the Green-Naghdi system provided the velocity in the Hamiltonian was approximated further to be columnar.

Finally, a new variational approach to analyse systems with discontinuities was explored. It resulted into known jump conditions for the shallow water system and novel conditions for our new system. Moreover, it provides an apparatus to analyse the stability of shocks or jumps for systems with the Hamiltonian structure. We were, however, unable to determine yet whether the jumps derived for the new system and its Boussinesq simplifications could be sustained via local dissipation of energy in the bore. Future plans therefore include the numerical evaluation of these jump conditions including shock-fitting methods, in contrast to shock-capturing methods in which artificial, numerical dispersion may incorrectly lead to smoothening of flows with discontinuities or bores.

\section{Appendix A}

\section{Integrals}

In this Appendix, we define the integrals in expressions (46)(50), as follows:

$$
\begin{array}{ll}
F(h, b)=\int_{b}^{b+h} f^{2} \mathrm{~d} z, & G(h, b)=\int_{b}^{b+h}\left(\partial_{h} f\right)^{2} \mathrm{~d} z, \\
K(h, b)=\int_{b}^{b+h}\left(\partial_{z} f\right)^{2} \mathrm{~d} z, & P(h, b)=\int_{b}^{b+h} f \mathrm{~d} z, \\
Q(h, b)=\int_{b}^{b+h}\left(\partial_{h} f\right) \mathrm{d} z, & R(h, b)=\int_{b}^{b+h} f\left(\partial_{h} f\right) \mathrm{d} z .
\end{array}
$$

When $f(z ; b, h)$ equals the parabolic vertical profile $(51)$, these integrals reduce to

$$
\begin{aligned}
& F=\frac{2}{15} h^{3}, \quad G=\frac{7}{15} h, \quad K=\frac{1}{3} h, \\
& P=-\frac{1}{3} h^{2}, \quad Q=-\frac{2}{3} h, \quad R=\frac{1}{5} h^{2} .
\end{aligned}
$$

\section{Appendix B}

\section{Variational Reynolds' transport theorem}

To take the variations of an integral with boundaries depending on dynamic variables, we have to obtain a variational analogue of Reynolds' transport theorem. Consider a domain $\Omega\left(x_{\Gamma}, y_{\Gamma}, z_{\Gamma}, t\right)$ in which (part of) the boundary $\partial \Omega_{\Gamma}$ is evolving in time. We need to find the variations of the integral

$$
I\left[F, \mathbf{x}_{\Gamma}\right]=\int_{\Omega\left(\mathbf{x}_{\Gamma}\right)} F(x, y, z, t) \mathrm{d} x \mathrm{~d} y \mathrm{~d} z,
$$

in which $F$ can depend implicitly on spatial coordinates and time via other variables, or an integral thereof, as in (76), or explicitly on $x, y, z$ and $t$. The variation has to be taken with respect to the function $F$ (in short, as in our case $F$ includes further dependencies) and the boundary positions $\mathbf{x}_{\Gamma}=\left(x_{\Gamma}, y_{\Gamma}, z_{\Gamma}\right)^{T}$. Taking a short-cut, the definition of the variation is

$\delta I=\lim _{\epsilon \rightarrow 0} \frac{1}{\epsilon}\left(I\left[F+\epsilon \delta F, \mathbf{x}_{\Gamma}+\epsilon \delta \mathbf{x}_{\Gamma}\right]-I\left[F, \mathbf{x}_{\Gamma}\right]\right)$.

We introduce a transformation $x=\chi(\xi, \eta, \zeta)$ (in short $\chi)$ from reference space to physical space with coordinates $\xi_{1}=\xi, \xi_{2}=\eta$ and $\xi_{3}=\zeta$ in the reference space. We assume that such a transformation (or compound of transformations) $\chi: \hat{\Omega} \mapsto \Omega$ exists. The evaluation of $F$ in the reference space is denoted by $F \circ \chi \equiv F(\mathbf{x}=\chi(\xi, \eta, \zeta), t)$, which includes the complicated dependence on the variables, as discussed. The inverse of $\chi$ is denoted by $\chi^{-1}$ and is assumed to exist. It transforms the physical domain $\Omega$ into a reference domain $\hat{\Omega}$. The key simplification used is that the reference domain is fixed in time. We denote the Jacobian matrix of this transformation by

$J=\left(\begin{array}{lll}x_{\xi} & x_{\eta} & x_{\zeta} \\ y_{\xi} & y_{\eta} & y_{\zeta} \\ z_{\xi} & z_{\eta} & z_{\zeta}\end{array}\right)$

and its determinant as $|J|$. Clearly, this Jacobian and the transformed coordinates will depend on the coordinates $\mathbf{x}_{\Gamma}$ along the boundary $\partial \Omega_{\Gamma}$.

The integral over the domain $\Omega$ is calculated to be

$\int_{\Omega} F(x, y, z, t) \mathrm{d} x \mathrm{~d} y \mathrm{~d} z=\int_{\hat{\Omega}} F \circ \chi|J| \mathrm{d} \xi_{1} \mathrm{~d} \xi_{2} \mathrm{~d} \xi_{3}$.

The variations are easier in reference space, given that the reference domain is fixed in time. We thus obtain

$$
\begin{aligned}
& \delta \int_{\hat{\Omega}} F \circ \chi|J| \mathrm{d} \xi_{1} \mathrm{~d} \xi_{2} \mathrm{~d} \xi_{3}=\int_{\hat{\Omega}} \delta(F \circ \chi|J|) \mathrm{d} \xi_{1} \mathrm{~d} \xi_{2} \mathrm{~d} \xi_{3} \\
= & \int_{\hat{\Omega}}|J| \delta(F \circ \chi)+(F \circ \chi) \delta|J| \mathrm{d} \xi_{1} \mathrm{~d} \xi_{2} \mathrm{~d} \xi_{3} .
\end{aligned}
$$


We consider the terms in (B5) consecutively. The first one becomes

$$
\int_{\hat{\Omega}}|J| \delta(F \circ \chi) \mathrm{d} \xi_{1} \mathrm{~d} \xi_{2} \mathrm{~d} \xi_{3}=\int_{\Omega} \delta(F \circ \chi) \circ \chi^{-1} \mathrm{~d} x \mathrm{~d} y \mathrm{~d} z .
$$

To evaluate the second term in (B5), we have to take variations of the Jacobian, as follows:

$\delta|J|=\nabla_{\xi} \cdot\left(|J|\left(J^{T}\right)^{-1} \mathrm{w}\right)$,

with the "variational wind" $\mathbf{w}=\delta \mathbf{x} \circ \chi$ denoting variations $\delta \mathbf{x}$ projected to the reference space, and $\nabla_{\xi} \equiv$ $(\partial / \partial \xi, \partial / \partial \eta, \partial / \partial \zeta)^{T}$. This leads to

$$
\begin{aligned}
\int_{\hat{\Omega}} & (F \circ \chi) \delta|J| \mathrm{d} \xi_{1} \mathrm{~d} \xi_{2} \mathrm{~d} \xi_{3} \\
= & \int_{\hat{\Omega}}(F \circ \chi) \nabla_{\xi} \cdot\left(|J|\left(J^{T}\right)^{-1} \mathrm{w}\right) \mathrm{d} \xi_{1} \mathrm{~d} \xi_{2} \mathrm{~d} \xi_{3} \\
= & \int_{\hat{\Omega}} \nabla_{\xi} \cdot\left((F \circ \chi)|J|\left(J^{T}\right)^{-1} \mathrm{w}\right) \\
& -\nabla_{\xi}(F \circ \chi) \cdot\left(|J|\left(J^{T}\right)^{-1} \mathrm{w}\right) \mathrm{d} \xi_{1} \mathrm{~d} \xi_{2} \mathrm{~d} \xi_{3} .
\end{aligned}
$$

It is worthwhile noting that we use the general vector $\delta \mathbf{x}$ because it depends through the transformation $\chi$, in a generally complicated manner, on $\delta \mathbf{x}_{\Gamma}$. The dependency does not need to be found explicitly as will become clear shortly. Only at the moving parts of the boundary $\partial \Omega_{\Gamma}$ is $\delta \mathbf{x}=\delta \mathbf{x}_{\Gamma}$, in contrast to the situation at the other boundaries.

In (B8), we consider the terms separately. The first one can be evaluated using Gauss' theorem as follows:

$$
\begin{aligned}
\int_{\hat{\Omega}} & \nabla_{\xi} \cdot\left((F \circ \chi)|J|\left(J^{T}\right)^{-1} \mathrm{~W}\right) \mathrm{d} \xi_{1} \mathrm{~d} \xi_{2} \mathrm{~d} \xi_{3} \\
& =\int_{\partial \hat{\Omega}}\left((F \circ \chi)|J|\left(J^{T}\right)^{-1} \mathrm{~W}\right) \cdot \boldsymbol{n}_{0} \mathrm{~d} \Gamma_{0} \\
& =\int_{\partial \Omega} F \delta \mathbf{x} \cdot \boldsymbol{n} \mathrm{d} \Gamma
\end{aligned}
$$

with $\boldsymbol{n}_{0}, \mathrm{~d} \Gamma_{0}$ and $\boldsymbol{n}, \mathrm{d} \Gamma$ the normal and line elements along the boundary in the reference and physical spaces, respectively. We note that $J^{T} \boldsymbol{n}=|J| \boldsymbol{n}_{0}$ follows directly from taking the gradient $\nabla_{\xi}$ of the equations of the same plane tangent to the surface at the boundary: $\boldsymbol{n} \cdot \mathbf{x}=C_{0}$ and $\boldsymbol{n}_{0} \cdot \boldsymbol{\xi}=C_{1}$ in the physical and references spaces. The actual constants $C_{0}$ and $C_{1}$ are unimportant and the determinant acts as a normalization.
The last term of (B8) is evaluated by using

$$
\nabla F=\left(J^{T}\right)^{-1} \nabla_{\xi}(F \circ \chi),
$$

such that we obtain

$$
\begin{aligned}
\int_{\hat{\Omega}} \nabla_{\xi}(F \circ \chi) \cdot\left(|J|\left(J^{T}\right)^{-1} \mathbf{W}\right) \mathrm{d} \xi_{1} \mathrm{~d} \xi_{2} \mathrm{~d} \xi_{3} \\
\quad=\int_{\Omega} \nabla F \cdot \delta \mathbf{x} \mathrm{d} x \mathrm{~d} y \mathrm{~d} z .
\end{aligned}
$$

After all, we combine the results in (B7) and (B11) into

$$
\begin{aligned}
& \delta \int_{\Omega} F(x, y, z, t) \mathrm{d} x \mathrm{~d} y \mathrm{~d} z=\int_{\partial \Omega} F \delta \mathbf{x} \cdot \boldsymbol{n} \mathrm{d} \Gamma \\
& \quad+\int_{\Omega} \delta(F \circ \chi) \circ \chi^{-1}-\nabla F \cdot \delta \mathbf{x} \mathrm{d} x \mathrm{~d} y \mathrm{~d} z .
\end{aligned}
$$

Using the chain rule for variations, one can derive that

$$
\int_{\Omega} \delta(F \circ \chi) \circ \chi^{-1} \mathrm{~d} x \mathrm{~d} y \mathrm{~d} z=\int_{\Omega} \nabla F \cdot \delta \mathbf{x}+(\delta F) \mathrm{d} x \mathrm{~d} y \mathrm{~d} z,
$$

such that we can combine the last two terms of (B12). Hence, we finally derived the required Reynolds' transport theorem for variations (80) used in the main text:

$$
\delta \int_{\Omega} F \mathrm{~d} x \mathrm{~d} y \mathrm{~d} z=\int_{\partial \Omega_{\Gamma}} F \delta \mathbf{x}_{\Gamma} \cdot \boldsymbol{n} \mathrm{d} \Gamma+\int_{\Omega} \delta F \mathrm{~d} x \mathrm{~d} y \mathrm{~d} z,
$$

where we used $\delta \mathbf{x}=\delta \mathbf{x}_{\Gamma}$, as $\delta \mathbf{x}=0$ on the fixed part of the boundaries.

\begin{abstract}
Acknowledgements. The authors gratefully acknowledge useful remarks from Vijaya R. Ambati regarding the shock relations. We thank co-authors Wout Zweers and Anthony Thornton (Bokhove et al., 2011) for support in using the displayed images of the bore-soliton-splash. We acknowledge financial support of the Netherlands Foundation for Technical Research (STW) for the project "Complex wave-current interactions in a numerical wave tank", and The Netherlands Organisation for Scientific Research (NWO) for the project "Compatible Mathematical Models for Coastal Hydrodynamics". A summary of part of the work was presented at a conference in Gagarina et al. (2012).
\end{abstract}

Edited by: R. Grimshaw

Reviewed by: two anonymous referees 


\section{References}

Ali, A. and Kalisch, H. : Energy balance for undular bores, C. R. Mecanique, 338, 67-70, doi:10.1016/j.crme.2010.02.003, 2010

Bokhove, O., Gagarina, E., Zweers, W., and Thornton, A.: BoreSoliton-Splash: van spektakel naar oceaangolf, Ned. Tijdschrift voor Natuurkunde, Popular version, 77, 450-454, available at: http://eprints.eemcs.utwente.nl/20683/ (last access: November 2011), 2011 (in Dutch).

Bonneton, P., Barthelemy, E., Chazel, F., Cienfuegos, R., Lannes, D., Marche, F., and Tissier, M.: Recent advances in SerreGreen Naghdi modelling for wave transformation, breaking and runup processes, Eur. J. Mech. B-Fluid.,30, 589-597, doi:10.1016/j.euromechflu.2011.02.005, 2011.

Boussinesq, J.: Théorie de l'intumescence liquide, applellée onde solitaire ou de translation, se propageant dans un canal rectangulaire, Comptes Rendus de l'Academie des Sciences, 72, 755759, 1871 (in French).

Bridges, T. J. and Needham, D. J.: Breakdown of the shallow water equations due to growth of the horizontal vorticity, J. Fluid Mech., 679, 655-666, doi:10.1017/jfm.2011.175, 2011.

Cotter, C. J. and Bokhove, O.: Variational water-wave model with accurate dispersion and vertical vorticity, J. Eng. Math., 67, 3354, doi:10.1007/s10665-009-9346-3, 2010.

Daniljuk, I. I.: On Integral Functionals with a Variable Domain of Integration, J. Proc. Steklov Inst. Math., 1-144, 1976.

Gagarina, E., van der Vegt, J. J. W., Ambati, V. R., and Bokhove, O.: A Hamiltonian Boussinesq model with horizontally sheared currents, in: Proc. 3rd Int. Symp. on Shallow Flows, June 4-6, 2012, Iowa, USA, available at: http://purl.utwente.nl/ publications/79724 (last access: 2012), 2012.

El, G. A., Grimshaw, R. H. J., and Smyth, N. F.: Unsteady undular bores in fully nonlinear shallow-water theory, Phys. Fluids, 18, 027104, doi:10.1063/1.2175152, 2006.

Flanders, H.: Differentiation under the integral sign, Am. Math. Mon., 80, 615-627, 1973.

Klopman, G., van Groesen, B., and Dingemans, M. W.: A variational approach to Boussinesq modelling of fully nonlinear water waves, J. Fluid Mech., 657, 36-63, doi:10.1017/S0022112010001345, 2010.
Kuznetsov, E. A. and Mikhailov, A. V. : On the topological meaning of canonical Clebsch variables, Phys. Lett., A 77, 37-38, doi:10.1016/0375-9601(80)90627-1, 1980.

Lin, C. C.: Liquid Helium, in: Proc. of the Enrico Fermi International School of Physics, Course XXI, Academic Press, New York, 93-146, 1963.

Luke, J. C. : A variational principle for a fluid with a free surface, J. Fluid Mech., 27, 395-397, doi:10.1017/S0022112067000412, 1967.

Miles, J. and Salmon, R.: Weakly dispersive nonlinear gravity waves, J. Fluid Mech., 157, 519-531, doi:10.1017/S0022112085002488, 1985.

Peregrine, D. H.: Surf zone currents, Theor. Comp. Fluid Dyn., 10, 295-310, doi:10.1007/s001620050065, 1998.

Peregrine, D. H. and Bokhove, O.: Vorticity and Surf Zone Currents, in: Proc. 26th Int. Conf. on Coastal Engineering 1998, ASCE, Copenhagen, 745-758, 1998.

Pratt, L. J.: On inertial flow over topography. Part 1. Semigeostrophic adjustment to an obstacle, J. Fluid Mech., 131, 195218, doi:10.1017/S0022112083001299, 1983.

Salmon, R.: Hamiltonian Fluid Mechanics, Annu. Rev. Fluid Mech., 20, 225-256, doi:10.1146/annurev.fl.20.010188.001301, 1988.

Shepherd, T. G.: A unified theory of available potential energy, Atmos. Ocean 31, 1-26, doi:10.1080/07055900.1993.9649460, 1993.

Wakelin, S. L.: Variational principles and the finite element method for channel flows, Ph.D. thesis, University of Reading, Reading, United Kingdom, 214 pp., 1993.

Whitham, G. B.: Linear and Nonlinear Waves, New York: Wiley, 635 pp., 1974

Yoshida, Z. : Clebsch parameterization: Basic properties and remarks on its applications, J. Math. Phys., 50, 113101, doi:10.1063/1.3256125, 2009.

Zweers, W.: Soliton-splash webpage with details of the boresoliton-splash experiment, youtube-videos and photos, available at: http://www.woutzweers.nl/, bore-soliton-splash youtube channel: http://www.youtube.com/user/woutzweers (last access: 2010), 2010 